\title{
Transfected plasmid DNA is incorporated into the nucleus via nuclear envelope reformation at telophase
}

Tokuko Haraguchi ( $\nabla$ haraguchi@fbs.osaka-u.ac.jp )

Osaka University https://orcid.org/0000-0002-3813-6785

\section{Takako Koujin}

National Institute of Communications Technology

\section{Tomoko Shindo}

Keio University

\section{Şükriye Bilir}

Osaka University

\section{Hiroko Osakada}

National Institute of Communications Technology

\section{Kohei Nishimura}

Nagoya University

\section{Yasuhiro Hirano}

Osaka university https://orcid.org/0000-0001-7339-4053

\section{Haruhiko Asakawa}

Osaka University https://orcid.org/0000-0001-9675-658X

\section{Chie Mori}

KARC, NICT

\section{Shouhei Kobayashi}

NICT

\section{Yasushi Okada}

RIKEN Quantitative Biology Center https://orcid.org/0000-0003-2601-3689

\section{Yuji Chikashige}

National Institute of Information and Communications Technology

\section{Tatsuo Fukagawa}

Osaka University https://orcid.org/0000-0001-8564-6852

\section{Shinsuke Shibata}

Keio University School of Medicine https://orcid.org/0000-0002-1185-9043

\section{Yasushi Hiraoka}

Osaka University https://orcid.org/0000-0001-9407-8228 
Article

Keywords: Transfection, nuclear envelope reformation, telophase, BAF, autophagy, CLEM, iCLEM

Posted Date: July 1st, 2021

DOI: https://doi.org/10.21203/rs.3.rs-478612/v1

License: (c) (i) This work is licensed under a Creative Commons Attribution 4.0 International License. Read Full License

Version of Record: A version of this preprint was published at Communications Biology on January 20th, 2022. See the published version at https://doi.org/10.1038/s42003-022-03021-8. 
1 Transfected plasmid DNA is incorporated into the nucleus via nuclear

\section{2 envelope reformation at telophase}

3

4 Tokuko Haraguchi ${ }^{1,2, *}$, Takako Koujin ${ }^{1,2}$, Tomoko Shindo ${ }^{3}$, Şükriye Bilir ${ }^{1,}$, Hiroko

5 Osakada $^{2}$, Kohei Nishimura ${ }^{1}$, Yasuhiro Hirano ${ }^{1}$, Haruhiko Asakawa $^{1}$, Chie Mori $^{2}$,

6 Shouhei Kobayashi ${ }^{2}$, Yasushi Okada ${ }^{4,5}$, Yuji Chikashige ${ }^{2}$, Tatsuo Fukagawa ${ }^{1}$, Shinsuke

$7 \quad$ Shibata $^{3,6}$, Yasushi Hiraoka ${ }^{1,2}$

8

$9 \quad{ }^{1}$ Graduate School of Frontier Biosciences, Osaka University, 1-3 Yamadaoka, Suita

10 565-0871, Japan

$11 \quad{ }^{2}$ Advanced ICT Research Institute Kobe, National Institute of Information and

12 Communications Technology, Kobe 651-2492 Japan

$13{ }^{3}$ Keio University School of Medicine, Shinjuku-ku, Tokyo 160-8582, Japan

14 4RIKEN Center for Biosystems Dynamics Research (BDR), Suita 565-0874, Japan

$15{ }^{5}$ Universal Biology Institute (UBI) and International Research Center for

16 Neurointelligence (IRCN), the University of Tokyo, Tokyo 113-0033, Japan

$17{ }^{6}$ Graduate School of Medical and Dental Sciences, Niigata University, Chuo-ku, Niigata

18 951-8510, Japan

19

20 *Corresponding author:

21 Tokuko Haraguchi

22 E-mail: haraguchi@fbs.osaka-u.ac.jp

23 Phone 81-6-6879-4621 
24 Keywords: Transfection, nuclear envelope reformation, telophase, BAF, autophagy,

25 CLEM, iCLEM

26 


\section{Abstract}

28 DNA transfection is an important technology in the life sciences, wherein nuclear entry 29 of DNA is necessary to achieve expression of the exogenous DNA. Non-viral vectors 30 and their transfection reagents are useful as safe tools for transfection. However, they

31 have no effects for transfection of non-proliferating cells, the reason for which remains

32 unknown. This study aimed to elucidate the mechanism by which transfected DNA

33 enters the nucleus for gene expression. To monitor the intracellular behavior of

34 transfected DNA, we introduced a plasmid bearing lac $O$ repeats and RFP-coding

35 sequences into cells expressing GFP-LacI and observed plasmid behavior and RFP

36 expression. RFP expression appeared only after mitosis. Electron microscopy showed

37 that plasmids were wrapped with nuclear envelope (NE)-like membranes or associated

38 with chromosomes at telophase. Depletion of BAF, which is involved in NE

39 reformation, delayed plasmid RFP expression. These results suggest that transfected

40 DNA is incorporated into the nucleus via NE reformation at telophase.

41 


\section{Introduction}

44 Transfection to introduce DNA plasmids into cells is an essential technology in the life

45 sciences and medical fields, for example, in gene therapy. Non-viral vectors and

46 transfection reagents, including cationic lipids or non-liposomal lipids, have been

47 developed as safe tools for introducing exogenous DNA into cells. DNA transfected

48 with these reagents is incorporated into the endosome via endocytosis and exposed to

49 the cytosol upon endosome rupture ${ }^{1-5}$, where it is detected by DNA sensor molecules ${ }^{6-}$

$50{ }^{10}$. However, it remains unclear how the transfected DNA in the cytosol enters the

51 nucleus. It is empirically known that DNA transfection, which leads to gene expression

52 from foreign DNA, is effective in proliferating cells but not in non-proliferating cells.

53 Therefore, it has been speculated that mitotic events, such as the nuclear envelope (NE)

54 breakdown, are important for nuclear entry of the transfected DNA. However, this has

55 not been experimentally proven yet.

56 Several attempts have been made to visualize the behavior of transfected DNA

57 and the cellular responses to $\mathrm{it}^{8,11,12}$. These include methods using fluorescent dye-

58 conjugated $\mathrm{DNA}^{11}$, the lacO/GFP-LacI visualizing system ${ }^{11}$, fluorescent nanodiamond

59 particles coated with DNA ${ }^{12}$, and beads conjugated with DNA (DNA-beads) ${ }^{8}$. In the

60 DNA-bead method, it has been shown that DNA-beads are internalized into cells via

61 endocytosis ${ }^{8,13}$, entrapped in endosomes, and exposed to the cytosol when the endosome

62 ruptures ${ }^{8}$. Endosome rupture stimulates autophagosome formation in the region of the

63 transfected DNA in a p62-dependent manner $^{14}$, leading to its degradation. However,

64 DNA on the beads simultaneously induces NE formation around the DNA-bead in a

65 barrier-to-autointegration factor (BAF)-dependent manner ${ }^{8}$. Once the NE forms around

66 the DNA beads, autophagy is suppressed ${ }^{8}$. 
67 BAF is a DNA binding protein ${ }^{15-18}$ involved in NE assembly ${ }^{19-21}$. It binds to

68 foreign DNA, such as transfected DNA and viral DNA, in the cytosol ${ }^{8,15,22-23}$, where it

69 assembles the NE-like membrane containing LEM domain proteins, such as emerin,

70 around the transfected $\mathrm{DNA}^{8}$. This BAF-dependent NE assembly is thought to occur

71 competitively with the assembly of the autophagy membrane ${ }^{8,24}$. However, the

72 biological significance of NE assembly on transfected DNA present in the cytosol is

73 unclear.

74 The NE is a double-membrane structure consisting of the inner and outer nuclear

75 membranes. The outer membrane is connected to the ER and contains proteins shared

76 with the ER, whereas the inner membrane contains transmembrane proteins specific to

77 the NE. Among the inner nuclear membrane proteins, LEM domain NE proteins,

78 including lamina-associated polypeptide 2 (Lap2), emerin, MAN1, and Lem2, are

79 highly conserved among metazoans ${ }^{25,26}$. LEM domain proteins bind BAF with the LEM

80 domain ${ }^{19,27-29}$. Beneath the inner nuclear membrane, there is a nuclear lamina consisting

81 of lamins ${ }^{30,31}$. There are fenestrated structures, called nuclear pores, spanning the

82 double-membrane structure of the $\mathrm{NE}^{32,33}$. The nuclear pore complex (NPC) is formed

83 at the pores. Nucleocytoplasmic transport between the nucleus and cytoplasm is carried

84 out through the NPC.

85 The NE is disassembled at the beginning of mitosis (prometaphase) and reformed

86 around chromosomes at the end of mitosis (telophase) in higher eukaryotic cells,

87 including human cells. During NE reformation at telophase, emerin and other LEM

88 domain proteins assemble at the "core region" of the chromosome mass in a BAF-

89 dependent manner, whereas lamin B receptor (LBR) and NPC components assemble in

90 the "non-core region" in a BAF-independent manner"19,21,34-36. The ESCRT-III protein 
91 complex functions to seal gaps in the nuclear membranes during NE reformation at 92 telophase ${ }^{37}$.

93 This study aimed to understand when and how transfected exogenous DNA enters

94 the nucleus. For this purpose, we transfected cells with DNA plasmids designed to

95 visualize the plasmids and monitor their expression. Here, we show that transfected

96 DNA is incorporated into the nucleus when the NE is being reformed during telophase.

97 Our findings will contribute to understanding how foreign DNA behaves within cells,

98 providing useful information for generating improved transfection reagents and

99 techniques.

100

$101 \quad$ Results

102 Protein expression from exogenous DNA occurs only after mitosis

103 To visualize transfected DNA and its expression, we generated the following

104 experimental system. We constructed a non-viral DNA plasmid (pLacO-pEF1 $\alpha$-mRFP)

105 carrying a lac $O$ repeat sequence (256 repeats, about $10 \mathrm{kbp}$ ) and a sequence expressing

106 monomeric red fluorescent protein (hereafter RFP) driven by the EF1 $\alpha$ promoter (Fig.

107 1a). We introduced it into human HeLa cells stably expressing EGFP-LacI (hereafter

108 GFP-LacI) using a non-liposomal lipid transfection reagent. Thus, when the DNA

109 plasmid enters the cytosol after endosome rupture, it is visualized by binding to GFP-

110 LacI, forming fluorescent puncta that are easily detected by fluorescence microscopy

111 (Fig. 1a; arrows, Fig. 1b). Plasmid that has not entered the cytosol cannot bind GFP-

112 LacI, and therefore will not be fluorescent (arrowheads, Fig. 1b).

We first assayed efficacy of the GFP-LacI signal to detect transfected

114 exogenous DNA in the cytosol. After removal of transfection reagents, cells were fixed 
and subjected to indirect immunofluorescence staining using anti-BAF. We examined

116 the overlap between the GFP-LacI signal and BAF, because it is known to bind

117 exogenous DNA immediately after it is released into the cytosol by endosome

118 rupture ${ }^{8,14,24}$. We found that the punctate cytoplasmic GFP-LacI signal, overlapped with

119 that of BAF (Fig. 1b), indicating that our system enabled the visualization of cytosolic

120 plasmid DNA. We also used the DNA-specific fluorescent dye, 4',6-diamidino-2-

121 phenylindole (DAPI), to confirm that the puncta contained DNA. Some of the DAPI

122 puncta did not colocalize with the GFP-LacI signal (arrowheads, upper panels, Fig. 1b),

123 suggesting that some plasmids were retained in endosomes. The GFP-LacI-positive

124 regions of DNA puncta colocalized with the LEM domain NE protein emerin (lower

125 panels, Fig. 1b), suggesting that they were surrounded by an NE-like membrane

126 immediately after release into the cytosol. This result is consistent with the report that

127 DNA-bead complexes become wrapped with NE-like membranes within 5-10 min after

128 endosome rupture ${ }^{8}$.

129 We then measured the timing of gene expression from transfected DNA by the

130 appearance of RFP fluorescence (Fig. 1c, d). Time-lapse analysis was carried out

131 immediately after transfection reagents were replaced with culture medium; images

132 were taken every $10 \mathrm{~min}$ for $18 \mathrm{~h}$ using DeltaVision or LSM880 microscopes. We

133 found that RFP signals appeared only in cells that had proceeded through mitosis (total

134 number of cells tested, $\mathrm{n}=594$ ) (Fig. 1c, d; Supplementary movies 1, 2), suggesting that

135 gene expression from the transfected plasmid does not occur during interphase before

136 mitosis. RFP expression was detected 2-5 $\mathrm{h}$ after the onset of mitotic chromosome

137 segregation (median $150 \mathrm{~min}, \mathrm{n}=594$ ) (Fig. 1d). Since the G1 phase lasts for $6-8 \mathrm{~h}$ in the

138 HeLa cell line ${ }^{38}$, our data suggest that gene expression occurs during the G1 phase in 
most cells (Fig. 1c, d). In these cells, the RFP signal appeared to be comparable in the two daughter cells. However, in a small fraction of the cells, RFP fluorescence appeared

141 later, after $500 \mathrm{~min}(6 \mathrm{~h} 20 \mathrm{~min})$. In these cells, the RFP signal often was confined to

142 only one of the daughter cells. The reason for this bias remains unknown. We also investigated gene expression from transfected DNA in U2OS cells, a

144 human osteosarcoma-derived cell line. We found that gene expression did not occur in

145 pre-mitotic cells, similar to HeLa cells. The timing of gene expression in U2OS cells

146 was 2-5 h (median $150 \mathrm{~min})$ after the onset of chromosome segregation $(\mathrm{n}=107)$

147 (Supplementary Fig. 1). This median value was the same as that obtained for HeLa

148 cells. These results indicate that gene expression from exogenous DNA transfected 149 using a non-viral transfection reagent occurs only after mitosis in these cell lines. Our 150 findings provide clear evidence for the first time that non-viral transfection is effective 151 only for proliferating cells.

\section{Dynamic behavior of exogenous DNA during mitosis}

154 Protein expression from plasmid DNA did not occur in some cells, even in the presence of GFP-LacI-positive clusters. To understand the difference in the behavior of plasmid DNA between cells with and without gene expression, we characterized the dynamic

157 behavior of GFP-LacI clusters during mitosis. Time-lapse analysis of GFP-LacI in

158 living mitotic cells revealed that in most, GFP-LacI clusters were disjointed at prometaphase with dispersion into smaller puncta during mitosis (Fig. 2a; intact during mitosis. Thus, we investigated whether this difference in mitotic behavior was related to gene expression. Most cells with GFP-LacI puncta that dispersed during 
mitosis expressed RFP during the subsequent interphase (62 out of 68 cells observed)

164 (Supplementary Fig. 2a), whereas the cells without dispersion did not (6 out of 68 cells)

165 (Supplementary Fig. 2b), suggesting that dispersion of plasmids may be important for

166 gene expression in the subsequent interphase.

167 We next investigated whether the presence or absence of NE was involved in this

168 GFP-LacI dispersion. Indirect immunofluorescence staining showed that GFP-LacI

169 puncta colocalized with emerin in interphase cells (lower panels, Fig. 1b). In

170 prometaphase cells, in which NE breakdown is ongoing, some of the GFP-LacI puncta

171 colocalized with emerin (arrowheads, upper panels in Fig. 2b), but others did not (lower

172 panels in Fig. 2b). Notably, small puncta (arrows, Fig. 2b') were found near the larger

173 puncta with no emerin signals (lower panels in Fig. 2b; Supplementary Fig. 2c),

174 suggesting that dispersion occurred upon NE breakdown. In metaphase cells, GFP-LacI

175 puncta did not colocalize with emerin (Fig. 2c). Many small puncta of GFP-LacI of the

176 same size were often found in metaphase cells (white puncta, Fig. 2d; Supplementary

177 movie 4). These results suggest that the dispersion of GFP-LacI puncta during mitosis is

178 caused by the breakdown of the NE-like membrane surrounding them. Fig. 2e

179 summarizes the behavior of the GFP-LacI puncta during mitosis.

180

181 Plasmid DNA is incorporated into the nucleus via NE reformation at telophase

182 As gene expression occurs only after mitosis (Fig. 1; Supplementary Fig. 1), we

183 hypothesized that plasmid DNA was incorporated into the nucleus via NE reformation

184 at the end of mitosis. In fact, small puncta of GFP-LacI, probably containing a single

185 copy or a few copies of plasmid DNA, were frequently observed near the telophase

186 chromosomes (arrows, Fig. 3a). To visualize the structural details of these GFP-LacI 
187 puncta, we performed correlative light and electron microscopy (CLEM) on telophase

188 cells undergoing NE reformation (Figs. 3b-f; Supplementary Fig. 3). We selected

189 telophase cells approximately 10 min after the onset of chromosome segregation for

190 CLEM analysis, in which most regions of the chromosomal mass were expected to be

191 surrounded by NE, although some regions may not yet be ${ }^{21}$. At the "core region" of the

192 chromosome mass, the NE containing LEM domain proteins is assembled in a BAF-

193 dependent manner, whereas in the "non-core region", the NE containing LBR and NPC

194 is assembled in a BAF-independent manner (right drawing in Fig. 3a) ) $^{19,21,36}$.

195 CLEM showed that puncta near the chromosomes at telophase were surrounded

196 by a double-membrane structure similar to the NE or endoplasmic reticulum (ER)

197 (labeled "dot 1" in Figs. 3b, c). This NE-like membrane was fused with the NE on the

198 chromosome (Fig. 3c), suggesting that the transfected DNA is incorporated into the

199 nucleus via membrane fusion. Multiple additional structures were found: dot 2 in Fig.

$2003 \mathrm{~d}$ was inside the nucleus (Fig. 3e), while dot 3 was outside and sandwiched between

201 two sets of ER or NE precursor membranes (Fig. 3e). Dot 4 in Fig. 3d was attached to

202 the chromosomes ("Type 4" in Fig. 3f; Supplementary Fig. 31-o). Relatively larger

203 puncta surrounded by ER or NE precursor membranes were also found in the cytoplasm

204 ("Type 1" in Fig. 3f; Supplementary Fig. 3a-d). We classified these puncta into five

205 types on the basis of their morphologies obtained by CLEM, as indicated in Fig. 3f (also

206 Supplementary Fig. 3): Type 1 (cytoplasmic circle) is a relatively large circular

207 structure covered with a double membrane, which is often found around the GFP-LacI

208 puncta present in the cytoplasm. Type 2 (sandwich) is an electron-dense structure

209 squeezed between two double membranes, suggestive of ER or NE precursor

210 membranes (Fig. 3e; Supplementary Fig. 3e-g). Type 3 (membrane fusion) is a structure 
211 in which a relatively large circular structure covered with a double membrane is fused

212 to the NE (Fig. 3c; Supplementary Fig. 3h-k). Type 4 (attached to chromosome) is a

213 structure at the edge of the chromosomal region where the NE is not present

214 (Supplementary Fig. 31-o). Type 5 (inside the nucleus) is a structure inside the nucleus

215 (between chromosomes) (Supplementary Fig. 3p-s). Besides these types, we frequently

216 observed large GFP-LacI puncta in the cytoplasm. These puncta were found in

217 degradative organelles, including lysosomes and autophagosomes.

218 To confirm the CLEM results, immuno-CLEM analysis was performed using an

219 anti-GFP antibody conjugated with Alexa and nanogold (Fig. 4). GFP-LacI puncta co-

220 stained with Alexa, locating near the telophase chromosome mass, were examined (Fig.

$2214 a, b$; arrows indicate dots 1-5). Dots 1 and 2 in Fig. 4 were located inside a relatively

222 large circle surrounded by a double membrane structure similar to NE or ER (Fig. 4c),

223 so were classified as type 1. Dot 3 was located on the edge or inside of the

224 chromosomal region (Fig. 4d) and classified as type 4 or 5 (compare with a typical

225 example of Types 4 and 5 in Supplementary Fig. 4). Dots 4 and 5 were located inside

226 the nucleus (Fig. 4e) and were classified as type 5 (compare with a typical example of

227 "Type 5" in Supplementary Fig. 4). These results suggest that at least part of the

228 transfected DNA is attached to the chromosome or located in the nucleus. Therefore,

229 our results suggest that plasmid DNA attaches telophase chromosomes and is

230 subsequently incorporated into the nucleus via NE reformation at the end of mitosis.

231 Fig. 4f shows the typical localization of GFP-LacI puncta at telophase.

232

233 BAF-dependent NE reformation at telophase facilitates nuclear entry 
234 To understand the molecular mechanism underlying nuclear entry of exogenous DNA in 235 telophase, we depleted NE proteins and examined the effect on gene expression from 236 the transfected DNA. BAF, emerin and Lem2 were selected as target proteins for 237 depletion because all are known to be involved in NE reformation during telophase ${ }^{21,39-}$

$238{ }^{41}$. We first employed the auxin-inducible degron (AID)-induced degradation system to 239 specifically deplete a target protein at the time of interest; for example, only during 240 mitosis $^{42,43}$. For this purpose, we integrated a sequence of mClover3-mAID into each of 241 the three target genes (BAFN1, EMD, and LEMD2) using CRISPR/Cas9 editing ${ }^{44}$. We 242 produced LEMD2 mutant cell lines (HeLa/mClover3-mAID-Lem2) expressing an 243 mClover3-mAID-Lem2 protein as the sole Lem2 protein (Supplementary Fig. 5), but 244 were not successful for BAF and emerin. Western blotting (WB) of lysates of cells 245 treated with indole-3-acetic acid (IAA) for 60 min showed that Lem2 was almost lost

246 (Supplementary Fig. 5b), consistent with the time-lapse microscopy data

247 (Supplementary Fig. 5c; Supplementary Movie 5). We examined the effect of Lem2 depletion on exogenous gene expression from the transfected plasmid using time-lapse microscopy (Fig. 5a). The fluorescence of the mClover3-mAID-Lem2 protein was almost completely lost in telophase ("IAA, 0:10" in

251 Fig. 5a) by the addition of IAA in metaphase ("IAA, -1:10" in Fig. 5a), suggesting that 252 depletion of Lem2 worked as expected. Time-lapse analysis of individual cells showed 253 that the timing of gene expression in Lem2-depleted cells was unchanged from that of 254 control cells (Fig. 5b), suggesting that Lem2 does not influence the expression of 255 exogenous DNA at telophase. Next, we used small interfering RNA (siRNA) treatment to deplete the target

257 proteins. HeLa cells were treated with siRNA against BAFN1 (iBAF, Fig. 5c-e), with 
siRNA against luciferase used as a control (iLuc, Fig. 5c-e). WB showed that the amount of BAF was reduced to $8 \%$ under these conditions (Fig. $5 \mathrm{~d}$ ). Then, cells depleted of BAF were transfected with the plasmid and gene expression was assessed in

261 individual cells by fluorescence microscopy (Fig. 5c). The timing of gene expression in cells treated with $B A F N 1$ siRNA (median 180 min, $\mathrm{n}=136$ ) was delayed relative to that of the control cells (median 140 min, n=129) (Fig. 5e). To confirm that BAF was truly depleted in the cells assayed by microscopy, a similar experiment was carried out using cells expressing GFP-BAF, which produced similar results (Supplementary Fig. 6a, b). Because BAF is known to be required for NE reformation at telophase ${ }^{21}$, these data suggest that NE reformation during telophase is an important step for the entry of exogenous DNA into the nucleus. We also tested the effect of emerin siRNA depletion, but it has no effect on the timing of gene expression (Supplementary Fig. 6c-e).

\section{NE rupture can cause pre-mitotic gene expression from exogenous DNA}

272 Our data led us to hypothesize that intact NE in interphase cells blocks the entry of foreign DNA into the nucleus. To test this hypothesis, we physically ruptured the NE,

274 then examined whether gene expression occurred from exogenous DNA without

275 telophase NE reformation. To do this, cells were transfected with plasmids.

276 Immediately after removal of the transfection reagent, the transfected cells were

277 microinjected with FITC-dextran to rupture the NE. FITC-dextran was used as a marker

278 for the NE rupture (Fig. 6a). Time-lapse microscopy showed that some of the NE-

279 ruptured cells expressed RFP from plasmid DNA, even in interphase cells that had

280 never undergone mitosis (Fig. 6b). Similarly, premitotic gene expression from

281 transfected DNA was observed in cells depleted of NE proteins. When emerin was 
282 depleted with siRNA treatment, premitotic gene expression from the transfected DNA

283 was observed (5 of 131 cells tested; Fig. 6c, d). No premitotic gene expression was

284 observed in the control siRNA treatment (129 cells tested; Supplementary Fig. 6e).

285 These results demonstrate that transient NE rupture caused by physical punctation or

286 depletion of the NE proteins allowed nuclear entry of the transfected DNA, resulting in

287 gene expression from foreign DNA, even in premitotic cells.

288

\section{Discussion}

290 Multiple cellular barriers to the delivery of DNA plasmids to the nucleus are known to

291 exist, including intracellular uptake, endosome acidification, autophagy, immune sensing pathways, and nuclear entry ${ }^{45,46}$. This study focused on how transfected DNA enters the nucleus. We found that gene expression occurs only after telophase, with

294 plasmids associated with telophase chromosomes enwrapped by the NE. Therefore, we concluded that NE reformation at telophase is important for the nuclear entry of the transfected DNA. This conclusion indicates that NE acts as a physical barrier to the delivery of foreign DNA into the nucleus, and that NPC-mediated nuclear transport of foreign DNA does not occur in interphase cells. If premitotic gene expression happened to occur in interphase cells, transient NE rupture might have been induced in these cells.

300 This hypothesis is consistent with our data showing that premitotic gene expression 301 occurs in cells in which the NE has been ruptured by a microinjection needle (Fig. 6a, b), and in cells in which emerin and BAF were depleted (Fig. 6c, d). Similar transient

303 NE ruptures have been reported in some diseases, including laminopathy and

304 cancers $^{47,48}$. In addition, BAF has been reported to play a role to repair NE rupture ${ }^{49}$, 305 consistent with our finding. 
307 endosomal escape, regardless of transfection method; moreover, the clusters are

308 relatively immobile during interphase ${ }^{11}$. The clusters remaining at metaphase are

309 asymmetrically partitioned with a bias to the younger centrosomes in dividing cells ${ }^{11}$.

310 We also found similar plasmid clusters in the cytosol. However, most of them were

311 dispersed at the beginning of mitosis to smaller puncta. The small puncta were scattered

312 throughout the cell and distributed to daughter cells (Fig. 2a; Supplementary movies 1

313 and 3), whereas the large puncta were retained in one cell as reported previously ${ }^{11}$.

314 Because gene expression from foreign DNA occurred only in cells with small puncta,

315 the small puncta that are incorporated into the reassembling nucleus at telophase are

316 associated with efficient gene expression from foreign DNA in the G1 phase. The

317 biological significance of plasmid clusters in mitosis remains unclear.

Transfected DNA in the cytosol activates DNA sensor signaling pathways ${ }^{6,7,50}$.

319 Binding of DNA sensors to the cytosolic DNA is hypothesized to be a defense response

320 against infection. Many DNA sensor proteins have been identified, including cyclic

321 GMP-AMP synthase (cGAS) $)^{51}$ and their common signaling adaptor stimulator of

322 interferon (IFN) gene (STING) ${ }^{52}$, DNA-dependent activator of interferon regulatory

323 factors/Z-DNA binding protein 1 (DAI/ZBP1) $)^{53}$, interferon activated gene $204(\mathrm{p} 204)^{54}$,

324 DEAD box helicase $60(\mathrm{DDX} 60)^{55}$, and $\mathrm{BAF}^{8,24}$. DAI/ZBP1 and p204 have been

325 reported to bind cytosolic plasmid DNA within 15 min, whereas DDX60 does not ${ }^{56}$.

326 BAF binds the cytosolic DNA within seconds ${ }^{8}$ and plays a role in assembling the NE-

327 like membrane around the plasmid DNA within approximately $10 \mathrm{~min}^{8}$. This BAF-

328 dependent NE assembly protects foreign DNA from autophagy ${ }^{8,24}$. Although BAF is

329 expected to function as a defense against viral infection ${ }^{23,57,58}$, it unexpectedly assists in 
the integration of the human immunodeficiency virus (HIV) genome into the host

331 genome ${ }^{15,16}$. In addition, a recent report showed that BAF protects against the cGAS-

332 STING response to prevent innate immune activation ${ }^{59,60}$. Collectively, these data

333 suggest that BAF can suppress the innate immune response and act to safely carry the

334 cytosolic DNA to the next cell cycle stages by wrapping it in the NE-like membrane.

335 In summary, our data suggest that NE acts as a barrier for foreign DNA to enter

336 the nucleus, and that foreign DNA is incorporated into the nucleus when the NE is

337 being reassembled in telophase. Our findings provide important insights not only for

338 understanding cellular responses during DNA transfection, but also for the development

339 of transfection reagents and methods.

$341 \quad$ Materials and Methods

342 Plasmids. We constructed the plasmid carrying a lac $O$ repeat sequence and a sequence

343 expressing RFP driven by the EF1 $\alpha$ promoter (pLacO-pEF1 $\alpha$-mRFP) as follows: First,

344 to generate the mRFP-C1 vector, the DNA fragment encoding mRFP was amplified by

345 PCR from the mRFP-pRSETB vector using the primer set mRFP-FWD3-Nhe (5'-

346 GAGCTAGCATGGCCTCCTCCGAGGACGTCA-3') and mRFP-REV2-Bgl (5'-

347 TAAGATCTGGCGCCGGTGGAGTGGCGGCCC-3'), and the PCR product was

348 inserted into the pEGFP-C1 vector (Clontech, Takara Bio Inc., Kusatsu, Japan) at the

349 NheI and BglII sites. The resulting plasmid is called the mRFP-C1 vector. Next, to

350 generate the pEF1 $\alpha$-mRFP plasmid, the DNA fragment encoding the EF1 $\alpha$-promoter

351 region was amplified by PCR from the pBOS-H2B-GFP vector (Stratagene, La Jolla,

352 CA) using the primers EF1 $\alpha$-prom.FWD-Ase (5'-

353 GGGATTAATCGTGAGGCTCCGGTGCCCGTCAGTGG-3') and EF1 $\alpha$-prom.REV- 
Nhe (5'-GGGCTAGCCTCACGACACCTGAAATGGAAGAAAAAAACTTTG-3'), and the PCR product was inserted into the mRFP-C1 vector at the AseI and NheI sites.

356 The resulting plasmid is called the $\mathrm{pEF} 1 \alpha-\mathrm{mRFP}$ plasmid. Then, the $\mathrm{pEF} 1 \alpha-\mathrm{mRFP}$

357 plasmid was digested with SalI, then circularized with a ligation kit (Ligation High

358 Ver.2, Toyobo Co., Osaka, Japan) after filling the gaps with E. coli DNA polI Klenow 359 fragment (Takara Bio Inc., Kusatsu, Japan) to generate the pEF1 $\alpha-\mathrm{mRFP}-\mathrm{C} 1-\Delta$ SalI 360 vector. The pEF1 $\alpha-m R F P-C 1-\Delta$ Sall vector was inserted with the sequence of

361 TAGTCGAC to generate a new SalI site in front of the EF1 $\alpha$ promoter region to

362 generate the SalI-pEF1 $\alpha-m R F P-C 1$ vector. Finally, to generate pLacO-pEF $1 \alpha-m R F P$,

363 the DNA fragment containing a $\mathrm{LacO}$-repeat sequence was obtained by digestion of the 364 pSV-dhfr DNA plasmid, then inserted into the SalI-pEF1 $\alpha$-mRFP-C1 vector at its SalI 365 site using Ligation High Ver.2 (Toyobo Co., Osaka, Japan). To construct the plasmid expressing EGFP-fused LacI (GFP-LacI), EGFP was

367 amplified by PCR from the pEGFP-C1 vector (Clontech, Takara Bio Inc., Kusatsu, 368 Japan) using the primers 5'-

369 TGTGACCGGGCGCCTACTATGGTGAGCAAGGGCGAGGAGCT-3' and 5'CGAATTCGCTAGCTCTAGACTTGTACAGCTCGTCCATGC-3', and the PCR

371 product was inserted into the PB-EF1 $\alpha$-MCS-IRES-Neo vector (PB533A-2, System

372 Biosciences, Mountain View, CA, USA) using XbaI sites to generate the PB-EF1 $\alpha$ -

373 EGFP-MCS-IRES-Neo vector. Next, DNA encoding LacI was amplified from the 374 MK9-38/pYC19 vector using the primers (5'-

375 ATTCGAATTTAAATCGGATATGGTGAAACCAGTAACGTTATA-3' and 5'376 CTCAGCGGCCGCGGATCTTACAGCTGCATTAATGAATCGGCC-3') and the 
377 PCR product was inserted into the PB-EF1 $\alpha$-EGFP-MCS-IRES-Neo vector using a

378 BamHI site to generate the plasmid expressing GFP-LacI.

379

380 Cell strains and culture. HeLa and U2OS cells were obtained from the Riken Cell

381 Bank (RCB007, Tsukuba, Japan) and the Health Protection Agency (London, UK), respectively. To generate HeLa cells stably expressing GFP-LacI (HeLa/GFP-LacI),

383 they were transfected with plasmids encoding GFP-LacI using Effectene (Qiagen,

384 Hilden, Germany), according to the manufacturer's instructions. Cells were selected

385 with $800 \mu \mathrm{g} / \mathrm{ml} \mathrm{Geneticin} \mathrm{(11811-031,} \mathrm{Life} \mathrm{Technologies,} \mathrm{Thermo} \mathrm{Fisher} \mathrm{Scientific,}$

386 Tokyo, Japan). Several clones were isolated from the survivors and their expression

387 levels were evaluated using WB. The selected clones were maintained in DMEM

388 containing 10\% fetal bovine serum (FBS) and $200 \mu \mathrm{g} / \mathrm{ml}$ Geneticin (11811-031, Life

389 Technologies, Thermo Fisher Scientific, Tokyo, Japan). We established LEMD2 mutant

390 cell lines (HeLa/mClover3-mAID-Lem2) expressing an mClover3-mAID-Lem2 protein

391 as the sole Lem2 protein as describe below (see section "Depletion of NE proteins" in

392 Materials and Methods).

393

394 Antibodies. Primary mouse monoclonal antibodies included anti-GFP for indirect

395 immunofluorescence staining and WB (Cat No. 632381, Lot No. 1004037, Clontech,

396 USA), anti- $\alpha$-tubulin (clone DM1A, Cat No. T9026, Lot No. 017H4838, Sigma), and

397 anti-BAF for WB (BANF1, Cat No. H00008815-M01, Lot No. 6132-S3, Abnova,

398 Taipei, Taiwan). Primary rabbit polyclonal antibodies included anti-GFP antibody for

399 iCLEM (Cat No. 600-401-215, Lot No. 24437, Rockland, Limerick, PA, USA), anti-

400 emerin (ED1) ${ }^{61}$, anti-BAF (PU38143) ${ }^{62}$, anti-Lem2 (LEMD2, Cat No. HPA017340, Lot 
402 Lot No. GR14272-3, Abcam). Alexa594-conjugated secondary antibodies against

403 mouse IgG (Cat No. A11037, Thermo Fisher Scientific) and rabbit IgG (Cat No.

404 A11032, Thermo Fisher Scientific) were used to detect the primary antibodies.

405

406 Transfection and live-cell imaging. HeLa/GFP-LacI cells were seeded on 35-mm

407 glass-bottom culture dishes (MatTek, MA, USA) at a concentration of $2 \times 10^{5}$ per dish

408 one day before imaging. For transfection, the cells were incubated for $4 \mathrm{~h}$ with

409 Effectene transfection reagent (Qiagen) containing $0.5 \mu \mathrm{g} /$ dish pLacO-pEF1 $\alpha-\mathrm{mRFP}$.

410 During this incubation, Hoechst 33342 was added to a final concentration of $100 \mathrm{ng} / \mathrm{ml}$

411 and incubated for 15 min to stain DNA. After transfection reagents were replaced with

412 culture medium, cells were subjected to time-lapse imaging. Images for each

413 wavelength were obtained every $10 \mathrm{~min}(1 \mu \mathrm{m} \times 7 \mathrm{z}$-stacks for a single time point

414 unless otherwise specified) through an oil-immersion objective lens UApo/340 (40x,

$415 \mathrm{NA}=1.35$, Olympus, Tokyo, Japan) using the DeltaVision fluorescence microscope

416 system (GE Healthcare Japan, Tokyo, Japan) in a temperature-controlled $\left(37^{\circ} \mathrm{C}\right)$ room;

417 this microscope system is equipped with a high-sensitive sCMOS camera (pico.edge

418 4.2, PCP AG, Kelheim, Germany). Images were also obtained every $10 \min (2 \mu \mathrm{m} \times 5$

419 z-stacks for a single time point) through a water-immersion objective lens C-Apo40

420 (40x, NA=1.2, Carl Zeiss, Jena, Germany) on a confocal fluorescence microscope

421 system LSM880 (Carl Zeiss), maintained at $37^{\circ} \mathrm{C}$ using a stage warmer. Scanning

422 conditions were as follows: pinhole size 2.5 A.U.; laser power 0.5-1\%; zoom 1; scan

423 speed 6; averaging 2 ; tiling $5 \times 5$. 
425 Immunofluorescence staining. HeLa/GFP-LacI cells were transfected with plasmid

426 using Effectene as described above. Immediately after removal of the transfection

427 reagent, cells were fixed with a fixative (3.7\% formaldehyde and $0.2 \%$ glutaraldehyde

428 in PBS) and incubated with anti-BAF or anti-emerin antibodies as previously

429 described $^{19,62}$. Anti-rabbit IgG-conjugated Alexa-594 was used as the secondary

430 antibody. Fluorescence images were obtained using an Olympus oil-immersion

431 objective lens PLAPON60xOSC $(\mathrm{NA}=1.40)$ on the DeltaVision microscope system.

432 Z-stacks of images (typically 20-30 focal planes at $0.5 \mu \mathrm{m}$ intervals) were obtained and

433 deconvoluted using software provided with the microscope system, SoftWorx.

434

435 Super-resolution microscopy. HeLa/GFP-LacI cells were transfected with plasmid

436 using Effectene as described above. After removal of the transfection reagent, cells

437 were fixed with a fixative (3.7\% formaldehyde and $0.2 \%$ glutaraldehyde in PBS). Then,

438 cells were observed by LSM880 Airyscan system (Carl Zeiss) equipped with Plan-

439 Apochromat 63x/1.40 M27 objective lens using immersion oil (\#518F, Carl Zeiss) at

440 room temperature (RT, approximately $26^{\circ} \mathrm{C}$ ). Pinhole size was set to $384 \mu \mathrm{m}$. Hoechst

441 and GFP were excited with 405 and $488 \mathrm{~nm}$ laser, respectively. The fluorescent signals

442 for Hoechst and GFP were collected using the following beam splitters and bandpass

443 filters: MBS-405 and BP 420-480 for Hoechst and MBS488/561 and BP 495-550 for

444 GFP. Images were acquired at 988 pixels each with $0.037 \mu \mathrm{m} /$ pixel. A z-stack image set

445 was acquired for 133 focal planes with $0.159 \mu \mathrm{m}$ intervals. After processing for super-

446 resolution image, brightness and contrast were changed for better visibility in Zen 2.3

447 SP1 software without changing gamma.

448 
449 Correlative light and electron microscopy (CLEM). HeLa/GFP-LacI cells were

450 transfected with plasmid using Effectene as described above. After removal of the

451 transfection reagent, cells were fixed with $2.5 \%$ glutaraldehyde for $1 \mathrm{~h}$. CLEM was

452 performed with some modifications of our previously published method ${ }^{21}$. Briefly, after

453 washing with PBS, the telophase cells 8-10 min after metaphase to anaphase transition

454 were selected by morphology, then subjected to fluorescence microscopy (FM) to

455 obtain 3D images (typically 40-60 focal planes at $0.2 \mu \mathrm{m}$ intervals) using a low-

456 chromatic-aberration oil-immersion lens PLAPON60xOSC $(\mathrm{NA}=1.40$, Olympus $)$ on a

457 DeltaVision microscope system. The images were deconvoluted to remove out-of-focus

458 images using software provided with the microscope system. Samples were post-fixed

459 with $1 \% \mathrm{OsO}_{4}(3002$, Nisshin EM, Tokyo, Japan), stained with $2 \%$ uranyl acetate

460 (8473-1M, Merck, Darmstadt, Germany), and then embedded in Epon812 (T024,

461 TAAB, England). Cells observed by FM were identified in the block. Ultrathin sections

462 of $80 \mathrm{~nm}$ were prepared and further stained with $4 \%$ uranyl acetate, followed by a

463 commercial ready-to-use solution of lead citrate (18-0875-2, Sigma-Aldrich, St. Louis,

464 MO, USA). Electron microscopy (EM) images were acquired using a JEM-1400

465 electron microscope ( $80 \mathrm{kV}$; JEOL, Tokyo, Japan). To correlate the FM and EM

466 images, we selected the FM image corresponding to the EM image from the 3D FM

467 images by eye using criteria including nuclear shapes and areas of bead cross-sections.

468

469 Immuno-CLEM (iCLEM). HeLa/GFP-LacI cells were transfected for $4 \mathrm{~h}$ with 0.2

$470 \mu \mathrm{g} /$ dish pLacO-pEF1 $\alpha-m R F P$ using Effectene as described above. After removal of

471 transfection reagents, cells were fixed with a mixture of $3.7 \%$ formaldehyde and $0.2 \%$

472 glutaraldehyde for 15 min. After washing with PBS, cells were permeabilized with 
$0.01 \%$ Triton $\mathrm{X}-100$ in PBS for 5 min at RT $\left(26^{\circ} \mathrm{C}\right)$, treated twice with $0.1 \%$ sodium

474 borohydride in PBS for 15 min, and washed twice with 1\% BSA in PBS for $1 \mathrm{~h}$. Cells

475 were then incubated with anti-GFP antibody (1:500 dilution in 1\% BSA in PBS, 600-

$476401-215$, lot. No. 35459, Rockland). After extensive washing with PBS (10 min $\times 12$

477 times), cells were incubated with a secondary Alexa594-nanogold-anti rabbit antibody

478 (1:200 dilution in 1\% BSA in PBS, Nanoprobes, Yaphank, NY, USA) for $3 \mathrm{~h}$ at RT. A

479 z-stack of fluorescence images (typically $40-60$ focal planes at $0.2-\mu \mathrm{m}$ intervals) were

480 acquired for cells of interest using a low-chromatic-aberration PLAPON60xOSC (NA =

481 1.40) objective on a DeltaVision microscope system as described above. Then, cells

482 were fixed with $1 \%$ glutaraldehyde for $1 \mathrm{~h}$ and washed with PBS three times. After

483 washing twice with $100 \mathrm{mM}$ Lysine-HCl/PB (pH 7.4) for $10 \mathrm{~min}$, cells were subjected

484 to silver enhancement as follows: cells were washed with PBS, incubated with $50 \mathrm{mM}$

485 HEPES (pH 5.8) three times, and washed with distilled water. Cells were then treated

486 with silver-enhancing reagent at $25^{\circ} \mathrm{C}$ for $5 \mathrm{~min}$ and washed with distilled water three

487 times. Samples were post-fixed with $1 \% \mathrm{OsO}_{4}$ (3002, Nisshin EM), stained with $2 \%$

488 uranyl acetate (8473-1M, Merck), and embedded in Epon812 (T024, TAAB) as

489 described previously ${ }^{21}$. Cells observed by fluorescence microscopy were identified in

490 the block. Ultrathin sections with a thickness of $80 \mathrm{~nm}$ were prepared and further

491 stained with 4\% uranyl acetate (8473-1M, Merck), followed by a commercial ready-to-

492 use solution of lead citrate (18-0875-2, Sigma-Aldrich). EM images were acquired

493 using a JEM-1400 plus electron microscope (80 kV; JEOL, Tokyo, Japan). To correlate

494 the FM and EM images, we selected FM image corresponding to EM images from the

495 3D FM images by eye, using criteria including nuclear shape and area of bead cross-

496 section in each image. 
498 Depletion of NE proteins. To deplete BAF, HeLa cells or HeLa cells stably expressing 499 GFP-BAF were treated with BAFN1 siRNA (Qiagen) using RNAiMax (Thermo Fisher 500 Scientific) as described previously ${ }^{21}$. To deplete emerin, HeLa cells were treated with $501 E M R$ siRNA [AACCGTGCTCCTGGGGCTGGG, Qiagen] ${ }^{63}$ using RNAiMax. Luciferase siRNA (Qiagen) was used as the negative control.

504 described previously ${ }^{43}$. The plasmid pAID1.2-EF1a-NmClover3-mAID, was developed 505 from pAID1.2-EF1a-NGFP-mAID (Addgene plasmid \#140607). The cDNA sequence 506 of Lem2 was amplified by PCR and cloned into the pAID1.2-EF1a-NmClover3-mAID 507 vector at the EcoRV site using the In-Fusion HD Cloning Kit (Takara Bio Inc.). The 508 pX330 plasmid targeting the human LEMD2 gene was constructed as described 509 previously $^{44}$. The sequence 5'-GACTTACTCACCAGCTTGGATGG-3' at the intron510 exon boundary of the 3' end of exon 3 of the human LEMD2 gene was selected as a 511 target for CRISPR/Cas9-mediated gene editing. These plasmids were cotransfected with 512 pAID EF1a linearized in pX330 (plasmid \#140610, Addgene) into HeLa cells. After 513 selection in $8 \mu \mathrm{g} / \mathrm{ml}$ blasticidin S (029-18701, Wako, Osaka, Japan), several clones 514 were picked; their expression levels of endogenous and mClover3-mAID-tagged Lem2 515 were evaluated by WB using anti-Lem 2 and anti-GFP antibodies, respectively. We used 516 an anti-GFP antibody to detect mClover3-mAID-Lem2 because the anti-GFP antibody 517 could cross-react with mClover3. AID-mediated protein degradation was induced by 518 adding final $0.5 \mathrm{mM}$ indole-3-acetic acid (IAA). 
520 Western Blotting (WB). WB was performed as previously described ${ }^{62}$. Briefly, cells

521 were collected and lysed in ice-cold isotonic buffer (20 mM Tris-HCl, pH 7.5, $2 \mathrm{mM}$

$522 \mathrm{MgCl}_{2}, 150 \mathrm{mM} \mathrm{NaCl}, 0.5 \% \mathrm{NP}-40$, Protease Inhibitor Cocktail) (final $1 \times 10^{7}$

523 cell $/ \mathrm{ml}$ ). Equal amounts of 2x SDS-PAGE sample buffer (without DTT) were added

524 to the lysates. After sonication, the lysates were boiled for $5 \mathrm{~min}$. DTT (freshly

525 prepared from powder) was added to the samples at a final concentration of $20 \mathrm{mM}$.

526 Ten micrograms of total protein from the cell extract were loaded into each lane of an

527 SDS 10-20\% gradient polyacrylamide gel or 15\% polyacrylamide gel. After

528 electrophoresis, proteins were transferred to PVDF membranes using a semi-dry

529 blotting system (Atto Corp., Tokyo, Japan) and stained using the ECL western blotting

530 detection system (GE Healthcare).

531

532 NE rupture. HeLa/GFP-LacI cells were transfected using the transfection reagent

533 Effectene (Qiagen) with $0.3 \mu \mathrm{g} /$ dish of pLacO-pEF1 $\alpha$-mRFP for $3 \mathrm{~h}$. During this

534 incubation, Hoechst 33342 was added to a final concentration of $100 \mathrm{ng} / \mathrm{ml}$ and

535 incubated for 15 min to stain DNA. After removal of the transfection mixture, the

536 culture medium was replaced with fresh DMEM containing 10\% FBS and then

537 subjected to fluorescence imaging. Cells in the glass-bottom culture dish were placed on

538 a stage of the DeltaVision fluorescence microscope in a temperature-controlled room

$539\left(37^{\circ} \mathrm{C}\right)$. Cells of interest, i.e., the cells appearing to be undergoing G1 phase, were

540 selected based on the morphology observed via bright-field microscopy; their NEs were

541 disrupted using the tip of a microinjection needle (Femtotips, Eppendorf, No.

5425242952008 Eppendorf, Hamburg, Germany) attached to a microinjector (microinjector

5435242 and micromanipulator 5171, Eppendorf). To confirm NE rupture, cells were 
544 concomitantly microinjected with $0.5 \mu \mathrm{g} / \mu \mathrm{l}$ FITC-dextran (MW, $10 \mathrm{kDa}$ ) as an injection

545 marker. Fluorescence images were obtained at 10-15 min intervals for $4 \mathrm{~h}$.

546

547 Data availability

548 The authors declare that the data supporting the findings of this study are available

549 within the paper and its supplementary information files. The source data underlying

550 Fig. 1d, 5b, 5e, Supplementary Fig. 1, 6b, and 6e are provided as a source data file.

551 Further data are available from the corresponding authors upon request.

552 


\section{References}

554 1. Conner, S. D., \& Schmid, S. L. Regulated portals of entry into the cell. Nature 422, 555 37-44. doi: 10.1038/nature01451 (2003).

556 2. Hoekstra, D., Rejman, J., Wasungu, L., Shi., F, \& Zuhorn, I. Gene delivery by

557 cationic lipids: in and out of an endosome. Biochem. Soc. Trans. 35, 68-71. doi:

$558 \quad 10.1042 / B S T 0350068(2007)$.

559 3. Zylberberg, C., Gaskil, K., Pasley, S., Matosevic, S. Engineering liposomal 560 nanoparticles for targeted gene therapy. Gene Ther. 24, 441-452. doi:

$561 \quad 10.1038 /$ gt.2017.41 (2017).

562 4. Brock DJ, Kondow-McConaghy HM, Hager EC, Pellois JP. Endosomal Escape and 563 Cytosolic Penetration of Macromolecules Mediated by Synthetic Delivery Agents.

564 Bioconjugate Chem. 30, 293-304. doi: 10.1021/acs.bioconjchem.8b00799 (2019).

565 5. Ita K. Polyplexes for gene and nucleic acid delivery: Progress and bottlenecks. Eur.

$566 \quad$ J. Pharm. Sci. 150, 105358. doi: 10.1016/j.ejps.2020.105358 (2020).

567 6. Takaoka, A., \& Taniguchi, T. Cytosolic DNA recognition for triggering innate 568 immune responses. Adv. Drug Deliv. Rev. 60, 847-57.

569 doi:10.1016/j.addr.2007.12.002 (2008).

570 7. Yanai, H., Savitsky, D., Tamura, T., \& Taniguchi, T. Regulation of the cytosolic 571 DNA-sensing system in innate immunity: a current view. Curr. Opin. Immunol. 21, 572 17-22. doi: 10.1016/j.coi.2009.01.005 (2009).

573 8. Kobayashi, S. et al. BAF is a cytosolic DNA sensor that leads to exogenous DNA 574 avoiding autophagy. Proc. Natl. Acad. Sci. USA 112, 7027-7032. doi:

$575 \quad$ 10.1073/pnas.1501235112(2015). 
9. Chen, Q., Sun, L., \& Chen, Z. J. Regulation and function of the cGASSTING pathway of cytosolic DNA sensing. Nat. Immunol. 17, 1142-9. doi: 10.1038/ni.3558 (2016).

10. Hopfner, K. P., \& Hornung, V. Molecular mechanisms and cellular functions of cGAS-STING signalling. Nat. Rev. Mol. Cell Biol. 21, 501-521. doi: 10.1038/s41580-020-0244-x (2020).

11. Wang, X., Le, N., Denoth-Lippuner, A., Barral, Y., \& Kroschewski, R. Asymmetric partitioning of transfected DNA during mammalian cell division. Proc. Natl. Acad. Sci. USA 113, 7177-82. doi: 10.1073/pnas.1606091113 (2016).

12. Petrakova, V., Benson, V., Buncek, M., Fiserova, A., Ledvina, M., Stursa, J., Cigler, P., \& Nesladek, M. I Imaging of transfection and intracellular release of intact, non-labeled DNA using fluorescent nanodiamonds. Nanoscale 8, 12002-12. doi: 10.1039/c6nr00610h.PMID: 27240633 (2016).

13. Kobayashi, S., Kojidani, T., Osakada, H., Yamamoto, A., Yoshimori, T., Hiraoka, Y., \& Haraguchi, T. Artificial induction of autophagy around polystyrene beads in nonphagocytic cells. Autophagy 6, 36-45 (2010).

14. Tsuchiya, M., Ogawa, H., Koujin, K., Kobayashi, S., Mori, C., Hiraoka, Y., \& $10.1002 / 1873-3468.12262(2016)$

15. Lee, M. S., \& Craigie, R. A previously unidentified host protein protects retroviral DNA from autointegration. Proc. Natl. Acad. Sci. USA 95, 1528-1533. doi:10.1073/pnas.95.4.1528 (1998). 
16. Chen, H. \& Engelman, A. The barrier-to-autointegration protein is a host factor for HIV type 1 integration. Proc. Natl. Acad. Sci. USA 95, 15270-15274. doi:10.1073/pnas.95.26.15270 (1998).

17. Umland, T. C., Wei, S.-Q., Craigie, R. \& Davies, D. R. Structural basis of DNA bridging by barrier-to-autointegration factor. Biochemistry 39, 9130-9138. doi:10.1021/bi000572w (2000).

18. Zheng, R., Ghirlando, R., Lee, M. S., Mizuuchi, K., Krause, M. \& Craigie, R. Barrier-to-autointegration factor (BAF) bridges DNA in a discrete, higher order

19. Haraguchi, T., Koujin, T., Segura-Totten, M., Lee, K. K., Matsuoka, Y., Yoneda, Y., Wilson, K. L., \& Hiraoka, Y. BAF is required for emerin assembly into the reforming nuclear envelope. J. Cell Sci. 114, 4575-4585 (2001).

612 20. Gorjánácz, M., Klerkx, E. P., Galy, V., Santarella, R., López-Iglesias, C., Askjaer, 613 P. \& Mattaj, I. W. Caenorhabditis elegans BAF-1 and its kinase VRK-1 participate 614 directly in post-mitotic nuclear envelope assembly. EMBO J. 26, 132-143.

615 doi:10.1038/sj.emboj.7601470 (2007).

616 21. Haraguchi, T., Kojidani, T., Koujin, T., Shimi, T., Osakada, H., Mori, C.,

617 Yamamoto, A., \& Hiraoka, Y. Live cell imaging and electron microscopy revealed 618 dynamic processes of BAF-directing nuclear envelope assembly. J. Cell Sci. 121, 619 2540-2554. doi: $10.1242 /$ jcs. 033597 (2008)

22. Wiebe, M. S., \& Traktman, P. Poxviral B1 kinase overcomes barrier to autointegration factor, a host defense against virus replication. Cell Host Microbe 1, 187-197. doi:10.1016/j.chom.2007.03.007 (2007). 
23. Ibrahim, N, Wicklund, A, Jamin, A, Wiebe, M. S. Barrier to autointegration factor (BAF) inhibits vaccinia virus intermediate transcription in the absence of the viral B1 kinase. Virology 444, 363-73. doi: 10.1016/j.virol.2013.07.002 (2013).

24. Kobayashi, S., \& Haraguchi, T. A novel pathway to detect and cope with exogenous dsDNA. Commun. Integr. Biol. 8, e1065361, doi:10.1080/19420889.2015.1065361 (2015).

25. Wagner, N., \& Krohne, G. LEM-domain proteins: new insights into lamininteracting proteins. Int. Rev. Cytol. 261, 1-46 (2007).

26. Brachner, A., \& Foisner, R. Evolvement of LEM proteins as chromatin tethers at the nuclear periphery. Biochem. Soc. Trans. 39, 1735-1741. doi:10.1042/BST20110724 (2011).

27. Cai, M., Huang, Y., Ghirlando, R., Wilson, K. L., Craigie, R., \& Clore, G.M. Solution structure of the constant region of nuclear envelope protein LAP2 reveals two LEM-domain structures: one binds BAF and the other binds DNA. EMBO J. 20, 4399-407. doi: 10.1093/emboj/20.16.4399 (2001).

28. Lee, K. K., Haraguchi, T., Lee, R. S., Koujin, T, Hiraoka, Y., \& Wilson, K. L. Distinct functional domains in emerin bind lamin A and DNA-bridging protein BAF. J. Cell Sci. 114, 4567-73 (2001).

29. Shumaker, D. K., Lee, K. K., Tanhehco, Y. C., Craigie, R., \& Wilson, K. L. LAP2 binds to BAF.DNA complexes: requirement for the LEM domain and modulation by variable regions. $E M B O J .20,1754-64$. doi: 10.1093/emboj/20.7.1754 (2001).

30. Moir, R. D., \& Goldman, R. D. Lamin dynamics. Curr. Opin. Cell Biol. 5, 408-11. doi: 10.1016/0955-0674(93)90004-a (1993). 
646

647

648

649

650

651

652

653

654

655

656

657

658

659

660

661

662

663

664

665

666

667

668

31. Tenga, R., \& Medalia, O. Structure and unique mechanical aspects of nuclear lamin filaments. Curr. Opin. Struct. Biol. 64, 152-159. doi: 10.1016/j.sbi.2020.06.017 (2020).

32. Hampoelz, B., Andres-Pons, A., Kastritis, P., \& Beck, M. Structure and Assembly of the Nuclear Pore Complex. Annu. Rev. Biophys. 48, 515-536. doi: 10.1146/annurev-biophys-052118-115308 (2019).

33. Bilir, Ş., Kojidani, T., Mori, C., Osakada, H., Kobayashi, S., Koujin, T., Hiraoka, Y., \& Haraguchi, T. Genes Cells 24, 338-353. doi: 10.1111/gtc.12677 (2019).

34. Shimi, T., Koujin, T., Segura-Totten, M., Wilson, K. L. Haraguchi, T. \& Hiraoka, Y. Dynamic interaction between BAF and emerin revealed by FRAP, FLIP and FRET analyses in living HeLa cells. J. Struct. Biol. 147, 31-41 (2004).

35. Dechat, T., Gajewski, A., Korbei, B., Gerlich, D., Daigle, N., Haraguchi, T., Furukawa, K., Ellenberg, J., \& Foisner, R. LAP2alpha and BAF transiently localize to telomeres and specific regions on chromatin during nuclear assembly. J. Cell Sci. 117, 6117-28. doi: 10.1242/jcs.01529. (2004).

36. Clever, M., Mimura, Y., Funakoshi, T., \& Imamoto, N. Regulation and coordination of nuclear envelope and nuclear pore complex assembly. Nucleus $\mathbf{4}$, 105-14. doi: 10.4161/nucl.23796 (2013).

37. Olmos, Y., Hodgson, L., Mantell, J., Verkade, P., \& Carlton, J.G. ESCRT-III controls nuclear envelope reformation. Nature 522, 236-9. doi: 10.1038/nature14503 (2015).

38. Haraguchi, T., Kaneda, T., \& Hiraoka, Y. Dynamics of chromosomes and microtubules visualized by multiple-wavelength fluorescence imaging in living 
mammalian cells: effects of mitotic inhibitors on cell cycle progression. Genes Cells 2, 369-80. doi:10.1046/j.1365-2443.1997.1280326.x (1997).

39. Asencio, C., Davidson, I. F., Santarella-Mellwig, R., Ly-Hartig, T. B., Mall, M., 672 Wallenfang, M. R., Mattaj, I. W., \& Gorjánácz, M. Coordination of kinase and 673 phosphatase activities by Lem 4 enables nuclear envelope reassembly during mitosis. Cell 150, 22-35. doi: 10.1016/j.cell.2012.04.043 (2012). L., Ullman, K. S., \& Frost, A. LEM2 phase separation promotes ESCRT-mediated

41. Dubińska-Magiera, M., Kozioł K, Machowska M, Piekarowicz K, Filipczak D, Rzepecki R. Emerin Is Required for Proper Nucleus Reassembly after Mitosis: Implications for New Pathogenetic Mechanisms for Laminopathies Detected in EDMD1 Patients. Cells 8, 240. doi: 10.3390/cells8030240 (2019).

42. Nishimura, K., \& Fukagawa T. An efficient method to generate conditional knockout cell lines for essential genes by combination of auxin-inducible degron tag and CRISPR/Cas9. Chromosome Res. 25, 253-260. doi: 10.1007/s10577-0179559-7 (2017).

43. Nishimura, K., Yamada, T., Hagihara, S., Iwasaki, R., Uchida, N., Kamura, T., Takahashi, K., Torii, K. U. \& Fukagawa, T. A super-sensitive auxin-inducible degron system with an engineered auxin-TIR1 pair. Nucleic Acids Res. 48, e108. doi: 10.1093/nar/gkaa748 (2020). 
691

692

693

694

695

696

697

698

699

700

701

702

703

704

705

706

707

708

709

710

711

712

713

714

44. Pyzocha, N. K., Ran, F. A., Hsu, P. D., \& Zhang, F. RNA-guided genome editing of mammalian cells. Methods Mol. Biol. 1114, 269-277. doi: 10.1007/978-1-62703761-7_17 (2014).

45. Olden, B. R., Cheng, E., Cheng, Y., \& Pun, S. H. Identifying key barriers in cationic polymer gene delivery to human T cells. Biomater. Sci. 7, 789-797. doi: 10.1039/c8bm01262h (2019).

46. Nguyen, J., \& Szoka, F. C. Nucleic acid delivery: the missing pieces of the puzzle? Acc. Chem. Res. 45, 1153-62. doi: 10.1021/ar3000162 (2012).

47. Houthaeve, G., Robigins, J., Braeckmans, K., \& De Vos, W. H. Bypassing Border Control: Nuclear Envelope Rupture in Disease. Physiology (Bethesda) 33, 39-49. doi: 10.1152/physiol.00029.2017 (2018).

48. Lim, S., Quinton, R. J., \& Ganem, N. J. Nuclear envelope rupture drives genome instability in cancer. Mol. Biol. Cell 27, 3210-3213. doi: 10.1091/mbc.E16-02-0098 (2016).

49. Halfmann, C. T., Sears, R. M., Katiyar, A., Busselman, B. W., Aman, L. K., Zhang, Q., O'Bryan, C. S., Angelini, T. E., Lele, T. P., \& Roux, K. J. Repair of nuclear ruptures requires barrier-to-autointegration factor. J. Cell Biol. 218, 2136-2149. doi: 10.1083/jcb.201901116 (2019).

50. Chiu, Y. H., Macmillan, J. B., \& Chen, Z. J. RNA polymerase III detects cytosolic DNA and induces type I interferons through the RIG-I pathway. Cell 138, 576-91. doi: 10.1016/j.cell.2009.06.015 (2009)

51. Sun, L., Wu, J., Du, F., Chen, X., \& Chen, Z. J. Cyclic GMP-AMP synthase is a cytosolic DNA sensor that activates the type I interferon pathway. Science 339, 786-91. doi: 10.1126/science.1232458 (2013). 
52. Burdette, D. L., Monroe, K. M., Sotelo-Troha, K., Iwig, J. S., Eckert, B., Hyodo,

716 M., Hayakawa, Y., \& Vance, R. E. STING is a direct innate immune sensor of cyclic di-GMP. Nature 478, 515-8. doi: 10.1038/nature10429 (2011).

53. Takaoka, A., Wang, Z., Choi, M. K., Yanai, H., Negishi, H., Ban, T., Lu, Y., Miyagishi, M., Kodama, T., Honda, K., Ohba, Y., \& Taniguchi, T. DAI (DLM1/ZBP1) is a cytosolic DNA sensor and an activator of innate immune response. Nature 448, 501-5. doi: 10.1038/nature06013. (2007).

54. Liu, C., Wang, H., Zhao, Z., Yu, S., Lu, Y.B., Meyer, J., Chatterjee, G., Deschamps, S., Roe, B. A. \& Lengyel, P. MyoD-dependent induction during myoblast differentiation of p204, a protein also inducible by interferon. Mol. Cell Biol. 20, 7024-7036 (2000).

55. Miyashita, M., Oshiumi, H., Matsumoto, M., \& Seya, T. DDX60, a DEXD/H box helicase, is a novel antiviral factor promoting RIG-I-like receptor-mediated signaling. Mol. Cell Biol. 31, 3802-3819. doi: 10.1128/MCB.01368-10 (2011).

56. Semenova, N., Bosnjak, M., Markelc, B., Znidar, K., Cemazar, M., \& Heller, L. Multiple cytosolic DNA sensors bind plasmid DNA after transfection. Nucleic Acids Res. 47, 10235-10246. doi: 10.1093/nar/gkz768 (2019).

57. Sears, R. M., \& Roux, K. J. Diverse cellular functions of barrier-toautointegration factor and its roles in disease. J. Cell Sci. 133, jcs246546. doi: $10.1242 /$ jcs.246546 (2020).

58. Wiebe, M.S., \& Jamin, A. The Barrier to Autointegration Factor: Interlocking Antiviral Defense with Genome Maintenance. J. Virol. 90, 3806-3809. doi: 10.1128/JVI.00178-16 (2016). 
59. Ma, H., Qian, W., Bambouskova, M., Collins, P. L., Porter, S. I., Byrum, A. K., Zhang, R., Artyomov, M., Oltz, E. M., Mosammaparast, N., Miner, J. J., Diamond, M. S. Barrier-to-Autointegration Factor 1 Protects against a Basal cGAS-STING Response. mBio 11, e00136-20. doi: 10.1128/mBio.00136-20 (2020).

60. Guey, B., Wischnewski, M., Decout, A., Makasheva, K., Kaynak, M., Sakar, M. S., Fierz, B., \& Ablasser, A. BAF restricts cGAS on nuclear DNA to prevent innate immune activation. Science 369, 823-828. doi: 10.1126/science.aaw6421 (2020).

61. Yorifuji, H., Tadano Y, Tsuchiya Y, Ogawa M, Goto K, Umetani A, Asaka Y, Arahata K. Emerin, deficiency of which causes Emery-Dreifuss muscular dystrophy, is localized at the inner nuclear membrane. Neurogenetics $\mathbf{1}, 135-40$. doi: 10.1007/s100480050020 (1997).

62. Haraguchi, T., Koujin, T., Hayakawa, T., Kaneda, T., Tsutsumi, C., Imamoto, N., Akazawa, C., Sukegawa, J., Yoneda, Y., \& Hiraoka, Y. Live fluorescence imaging reveals early recruitment of emerin, LBR, RanBP2, and Nup153 to reforming functional nuclear envelopes. J. Cell Sci. 113, 779-794 (2000).

63. Harborth, J., Elbashir, S. M., Bechert, K., Tuschl, T., \& Weber, K. Identification of essential genes in cultured mammalian cells using small interfering RNAs. $J$ Cell Sci. 114, 4557-65. (2001).

\section{Acknowledgements}

We are grateful to Dr. Ritsuko Sahashi and Ms. Noriko Fukuta for technical assistance for experiments, Dr. Kentaro Kogure (Tokushima University, Japan) for insightful discussion, and Dr. Ruth Kroschewski (ETH Zürich, Switzerland) for critical reading of the manuscript. This work was supported by JST CREST JPMJCR15G2 to YO and 
762 following JSPS grants: JP17K19505 and JP18H05528 to TH; JP19K06611 to KN;

763 JP19H05795, JP19H03394, and JP16H06280 to YO: JP17H06167 and JP15H05972 to

764 TF; JP20H00454 and JP18H05533 to YHiraoka.

765

766 Author contributions

$767 \mathrm{TH}$, and YHiraoka conceived and designed the experiments. TK, TS, ŞB, HO, KN,

768 YHirano, CM, and SK performed experiments. TH, HA, YO, YC, TF, SS, and YHirano

769 analyzed the data. All the authors contributed reagents/materials/analysis tools. TH,

770 YHirano, HA and YHiraoka wrote the manuscript with the input from all the authors.

771

772 Competing interests

773 The authors declare that they have no competing interests in relation to this work.

774

775 Materials and Correspondence

776 Material request and correspondence should be sent to Tokuko Haraguchi (haraguchi@

777 fbs.osaka-u.ac.jp).

778

779 


\section{Figure legends}

781 Fig. 1. Gene expression from foreign DNA occurs after mitosis.

782 a. Schematic of the experiment. Top: diagram of pLacO-pEF1 $\alpha-m R F P$. This plasmid

783 carries a $l a c O$ repeat sequence (256 repeats, about $10 \mathrm{kbp}$ ) and a sequence expressing a

784 monomeric red fluorescent protein (RFP) under the EF1 $\alpha$ promoter. Bottom left: when

785 the transfected plasmids are exposed to the cytosol upon endosome rupture, it binds to

786 GFP-LacI and becomes fluorescent. Bottom right: when it enters the nucleus,

787 expression of RFP occurs. Therefore, RFP florescence is an indication of the nuclear

788 translocation of the plasmid. While using this system, gene expression was determined

789 by the appearance of RFP fluorescence.

790 b. HeLa/GFP-LacI cells transfected with pLacO-pEF1 $\alpha$-mRFP $(0.5 \mu \mathrm{g} / \mathrm{dish})$

791 diagrammed in panel a. Immediately after removal of the transfection reagent, cells

792 were fixed and immuno-stained using anti-BAF or anti-emerin antibodies. DNA

793 (stained with DAPI, blue), GFP-LacI (green), and BAF (red) or emerin (red) in the

794 merged image. Arrows indicate DAPI signals with GFP-LacI signals. Arrowheads

795 indicate DAPI signals without GFP-LacI signals. The inset shows an image with

796 increased brightness in the boxed region. Bar, $10 \mu \mathrm{m}$

797 c. Time-lapse images of transfected HeLa/GFP-LacI cells. DNA was stained with

798 Hoechst 33342. Immediately after removal of the transfection reagent, time-lapse

799 images for each wavelength were acquired at 10-min intervals $(1 \mu \mathrm{m} \times 7 \mathrm{z}$-stacks for

800 each time-point) using DeltaVision. Maximum intensity projection images for z-stacks

801 for selected time-points are shown. The numbers represent time (h:min). Time 0

802 represents the onset of chromosome segregation. Black-and-white inverted images are 
804 (green), and RFP (red). Bar, $10 \mu \mathrm{m}$.

805 d. Timing of gene expression from transfected DNA. Timing of RFP expression was 806 determined for individual cells as shown in panel (c). Time 0 represents the onset of 807 chromosome segregation. Cell numbers tested were $n=248$ by DeltaVision and $n=346$ 808 by LSM880; total number, $n=594$. These data are combined in this graph. M, G1, and S 809 represent the cell cycle phases estimated based on our previous report ${ }^{38}$.

\section{Fig. 2. DNA puncta disperse during mitosis.}

812 a. Representative time-lapse images of HeLa/GFP-LacI cells transfected with pLacO813 pEF1 $\alpha$-mRFP. DNA was stained with Hoechst 33342. Immediately after removal of the

814 transfection reagent, time-lapse images for each wavelength were acquired at 10-min 815 intervals $(1 \mu \mathrm{m} \times 7 \mathrm{z}$-stacks for each time-point $)$ using DeltaVision. The z-stack images 816 were deconvoluted. Maximum intensity projection images for the deconvoluted z-stacks 817 of selected time points are shown. Time 0 represents the leftmost image $(680 \mathrm{~min}$ after 818 the start of image acquisition). Black-and-white inverted images are shown for each 819 wavelength. Merged images, DNA (red) and GFP-LacI (green). Bar, $10 \mu \mathrm{m}$.

820 b. HeLa/GFP-LacI cells transfected with pLacO-pEF1 $\alpha$-mRFP, fixed and immuno-

821 stained for emerin as indicated in Fig. 1b. A single, representative prometaphase cell is

822 shown. The upper and lower rows show the same cell at focal planes $1.5 \mu \mathrm{m}$ apart.

823 Arrowheads indicate small puncta with emerin signals. Black-and-white inverted

824 images are shown for each wavelength. Merged images, DNA (DAPI, blue), GFP-LacI 825 (green), and emerin (red). Bars, $10 \mu \mathrm{m}$. 
827 puncta.

828 c. Same as panel $b$ except that a metaphase cell is shown. Bar, $10 \mu \mathrm{m}$.

829 d. Super-resolution images of GFP-LacI and chromosomes in a metaphase cell.

$830 \mathrm{HeLa} / \mathrm{GFP}-\mathrm{LacI}$ cells were transfected with $\mathrm{pLacO}-\mathrm{pEF} 1 \alpha-\mathrm{mRFP}$. After transfection,

831 cells were fixed with a fixative as described in Methods. Super-resolution images were

832 obtained using LSM880 Airyscan microscope. White and magenta represent small

833 puncta of transfected DNA and chromosomes, respectively. Bar, $10 \mu \mathrm{m}$.

834 e. Diagrams of transfected DNA in interphase, prometaphase, and metaphase cells.

835

836

Fig. 3. CLEM images showing small puncta of transfected DNA in telophase cells.

$837 \mathrm{HeLa} / \mathrm{GFP}-\mathrm{LacI}$ cells were transfected with $\mathrm{pLacO}-\mathrm{pEF} 1 \alpha-\mathrm{mRFP}$, fixed and subjected

838 to indirect immunofluorescence imaging (a) or CLEM (b-f).

839 a. Indirect immunofluorescence images of telophase cells. After removal of the

840 transfection reagent, cells were fixed and immuno-stained for emerin. DAPI (DNA,

841 blue), GFP-LacI (green), emerin (red) are shown in the merged image. The rightmost

842 cartoon illustrates the core (red) and non-core regions (green) of the NE forming around

843 the telophase chromosomes. Arrows, positions of GFP-LacI. Bar, $10 \mu \mathrm{m}$.

844 b. Representative CLEM of telophase cells. Green and magenta represent GFP-LacI and

845 DAPI signals, respectively. Left and right, fluorescence and electron microscopic

846 images, respectively. Middle, merged image. Bar, $5 \mu \mathrm{m}$.

847 c. Electron micrographs of the yellow-boxed region of dot 1 in panel b. Arrows,

848 positions of GFP-LacI marked with green. Bar, $500 \mathrm{~nm}$. 
849 d. CLEM image. Different focal planes of the cell shown in panel (b). Left and right,

850 fluorescence and electron microscopic images, respectively. Middle, merged image.

851 Arrows, positions of GFP-LacI (dots 2-4). Bar, $5 \mu \mathrm{m}$.

852 e. Higher magnification of the red-boxed region in (d). Red circles represent the regions

853 of the GFP-LacI signals, marked in green. Bar, $500 \mathrm{~nm}$.

854 f. Classification of puncta morphologies. Type 1 (cytoplasmic circle): plasmid

855 contained within a circular structure surrounded by an NE-like membrane that is present

856 in the cytoplasm. Type 2 (sandwich): plasmid is present between NE-like membranes.

857 Type 3 (membrane fusion): plasmid contained in the cytoplasmic circle fused with the

858 NE. Type 4 (attached to chromosome): plasmid attached to the telophase chromosomes.

859 Dot 4 is the same "dot 4" shown in panel (d). Type 5 (inside the nucleus): plasmid

860 inside the nucleus. Middle panels, schematics of the electron microscopic images. NE is

861 shown as a double line. Red arrows indicate the positions of GFP-LacI, marked in

862 green. Electron microscope images of wider areas, including the area shown in this

863 figure, are shown in Supplementary Figure 3. Bars, $500 \mathrm{~nm}$ for Types $1-4$ and $1 \mu \mathrm{m}$ for 864 Type 5.

865

866 Fig. 4. Immuno-CLEM images of telophase cells.

$867 \mathrm{HeLa} / \mathrm{GFP}-\mathrm{LacI}$ cells were transfected with pLacO-pEF1 $\alpha$-mRFP, fixed and subjected 868 to immuno-CLEM.

869 a. Fluorescence images. The images on the left are overviews. DNA (stained with

870 Hoechst 33342, blue), GFP-LacI (green), and Alexa-nanogold (red) are shown in the

871 merged images. Right: enlarged image of the red-boxed region. Arrows 1-5 correspond

872 to the dots analyzed in panels b-e. 
873 b. Low-magnification electron micrograph of the telophase cell shown in panel (a).

874 Blue contour, telophase chromosomal region. The right image shows merged electron

875 and fluorescence micrographs. Arrows 1-5 indicate the positions analyzed; red-, blue-,

876 and yellow-boxed regions are enlarged in panels $\mathbf{c}, \mathbf{d}$, and $\mathbf{e}$, respectively. Bar, $2 \mu \mathrm{m}$.

877 c. The red-boxed region in panel b. Red lines, NE-like structures. Arrows, regions

878 where the signals are concentrated. Blue line, edge of the nucleus. Nuc, Cyt, and MT

879 label the nucleus, cytoplasm, and mitochondria, respectively. Bars, $500 \mathrm{~nm}$.

880 d. The blue-boxed region in panel b. Red lines, NE-like structures. Arrows, regions

881 where the signals are concentrated. Blue line, edge of the nucleus. Nuc and Cyt label the

882 nucleus and cytoplasm, respectively. Bars, $500 \mathrm{~nm}$.

883 e. The yellow-boxed region in panel b. Arrows, regions where the signals are

884 concentrated. Blue line, edge of the nucleus. Nuc and Cyt label the nucleus and

885 cytoplasm, respectively. Bars, $500 \mathrm{~nm}$.

886 f. Diagrams of typical localizations of transfected DNA in telophase cells. Types 1-5 are 887 classified in Fig. 3f.

888

889 Fig. 5. Depletion of BAF delays plasmid gene expression.

890 a. Time-lapse images of HeLa/mClover3-mAID-Lem2 cells. HeLa/mClover3-mAID-

891 Lem2 were transfected with pEF1 $\alpha$-mRFP plasmid. After removal of the transfection

892 reagent, time-lapse images for each wavelength were acquired every 10 or $15 \min (2$

$893 \mu \mathrm{m} \times 5 \mathrm{z}$-stacks for each time point) using DeltaVision. IAA (indole-3-acetic acid) or

894 ethanol as a control was added to HeLa/mClover3-mAID-Lem2 cells during live-cell

895 imaging. Maximum intensity projection images of selected time points are shown. The

896 numbers represent time (h:min). Time 0 is the onset of chromosome segregation. Black 
898 (green), RFP (magenta) and Hoechst 33342 (white). Orange arrows indicate timing of 899 RFP expression. Bar, $10 \mu \mathrm{m}$.

900 b. Timing of RFP expression in control parental (control) and Lem2-depleted HeLa 901 (IAA) cells. Timing of RFP expression was determined for individual cells as shown in 902 panel (a). Time 0 is the onset of chromosome segregation. Cell numbers tested are 903 indicated in the graph.

904 c. Time-lapse images of transfected HeLa cells treated with siRNA targeting BAFN1 905 (iBAF) and control siRNA (iLuc). Cells were treated with siRNA (iBAF or iLuc) and 906 transfected with pEF1 $\alpha$-mRFP plasmid. DNA was stained with Hoechst 33342 during 907 transfection. Immediately after removal of the transfection reagent, time-lapse images 908 for each wavelength were acquired at 10 -min intervals $(2 \mu \mathrm{m} \times 5 \mathrm{z}$-stacks for a single 909 time-point) using DeltaVision. Images projected by the maximum intensity projection 910 for z-stacks are shown. RFP expression from transfected DNA and Hoechst 33342 are 911 shown in magenta and white, respectively. The numbers represent time (h:min). Time 0 912 represents the onset of chromosome segregation. Bar, $10 \mu \mathrm{m}$.

913 d. Western blotting of lysates of HeLa cells treated with siRNA targeting BAFN1

914 (iBAF) and control siRNA (iLuc). Tubulin was used as a loading control.

915 e. Timing of RFP expression in HeLa cells treated with iBAF and iLuc. Timing of RFP 916 expression was determined for individual cells as shown in panel (c). Time 0 represents 917 the onset of chromosome segregation. Cell numbers tested are indicated in the graph. 918

919 Fig. 6. NE rupture causes premitotic gene expression. 
920 a. HeLa cells were transfected with pEF1 $\alpha$-mRFP plasmid. NE was ruptured with a

921 microinjection needle by microinjecting FITC-dextran into the nucleus. Left,

922 microinjected cells (green) marked with FITC-dextran. Right, representative image of

923 microinjection needle used for NE rupture. Bar, $10 \mu \mathrm{m}$.

924 b. Time-lapse images of cells with NE rupture shown in (a). Immediately after NE

925 rupture, time-lapse images for each wavelength were captured every $10 \min (2 \mu \mathrm{m} \times 5$

$926 \quad$ z-stacks for a single time-point) using DeltaVision. Images projected by the maximum

927 intensity projection for z-stacks are shown. From the left, FITC-dextran, RFP, and

928 Hoechst images are shown. Black-and-white inverted images are shown for each

929 wavelength. Merged images (second column from the right), FITC-dextran (green),

930 RFP (red), and Hoechst 33342 (blue). The cell in the red-boxed region is shown in the

931 lower panels. The numbers represent time (h:min) from the start of imaging. Red arrow

932 indicates the start of RFP expression. G1, S, G2, and M represent the cell cycle phases

933 estimated based on our previous report ${ }^{38}$. Bars, $10 \mu \mathrm{m}$.

934 c. Western blotting of HeLa cells treated with siRNA targeting EMD (iemerin) and the

935 control (iLuc), showing that emerin expression in the cells treated with siRNA was

936 reduced to $17 \%$ of control levels.

937 d. Time-lapse images of the cells treated with iemerin. HeLa cells were treated with

938 siRNA targeting EMD (iemerin). The cells were transfected with pEF1 $\alpha-\mathrm{mRFP}$

939 plasmid. After transfection reagent was replaced with the culture medium, time-lapse

940 images for each wavelength were collected every $10 \min (2 \mu \mathrm{m} \times 5 \mathrm{z}$-stacks for a single

941 time-point) using DeltaVision. Images projected by the maximum intensity projection

942 for z-stacks are shown. The numbers represent time (h:min) from the start of imaging.

943 Expression of RFP started in pre-mitotic cells (white arrow). White arrow indicates the 
944 start of RFP expression. S, G2 and M represent the S, G2 and M phases of the cell 945 cycle. Bar, $10 \mu \mathrm{m}$. 
a)
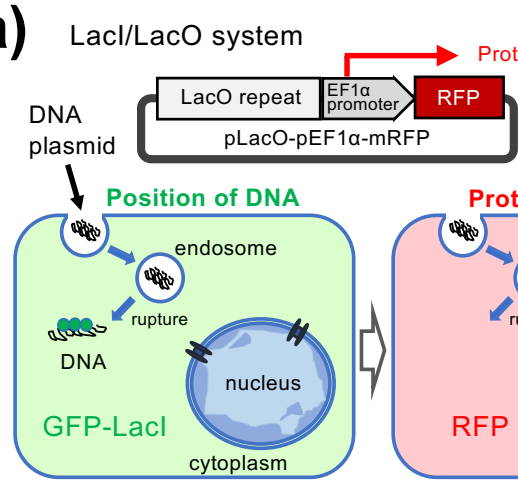

Protein expression endosome

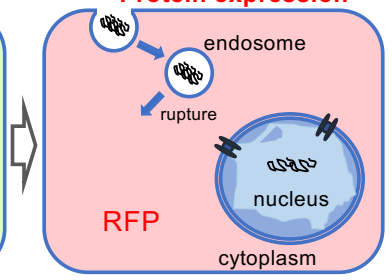

c)

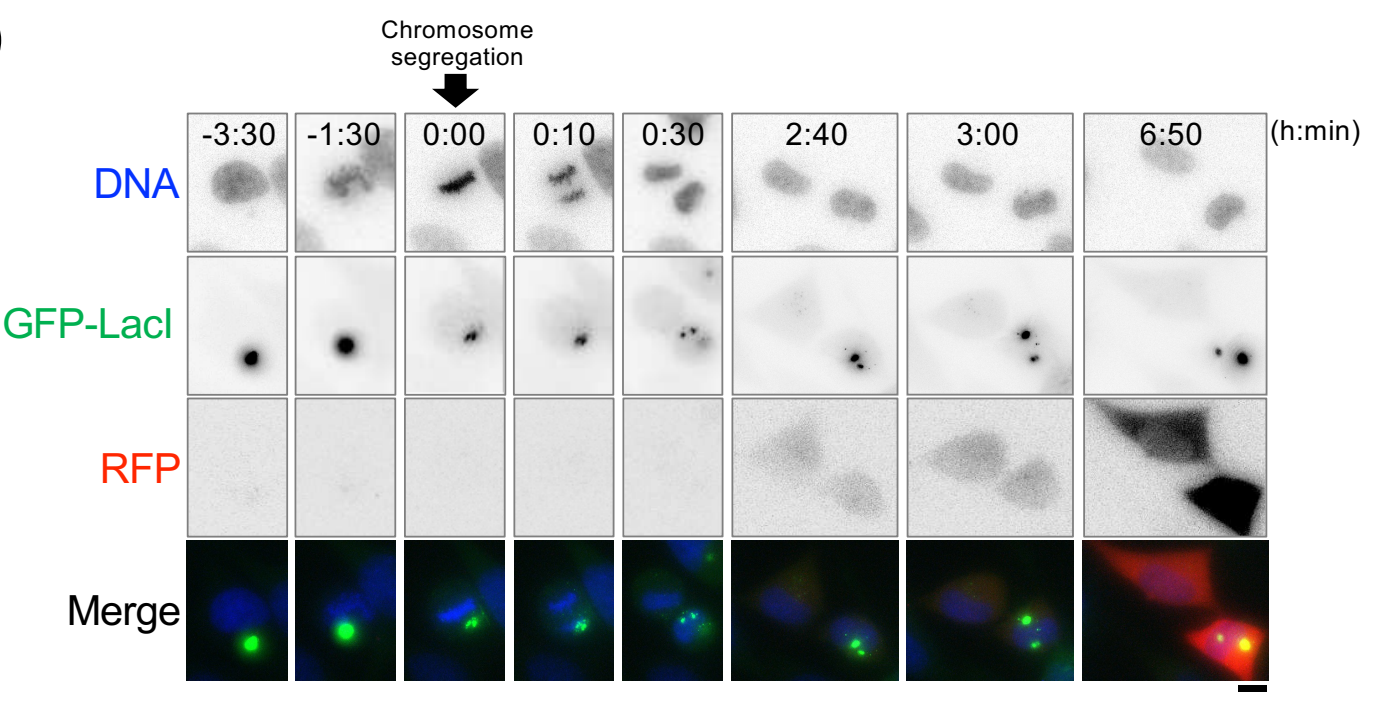

d)

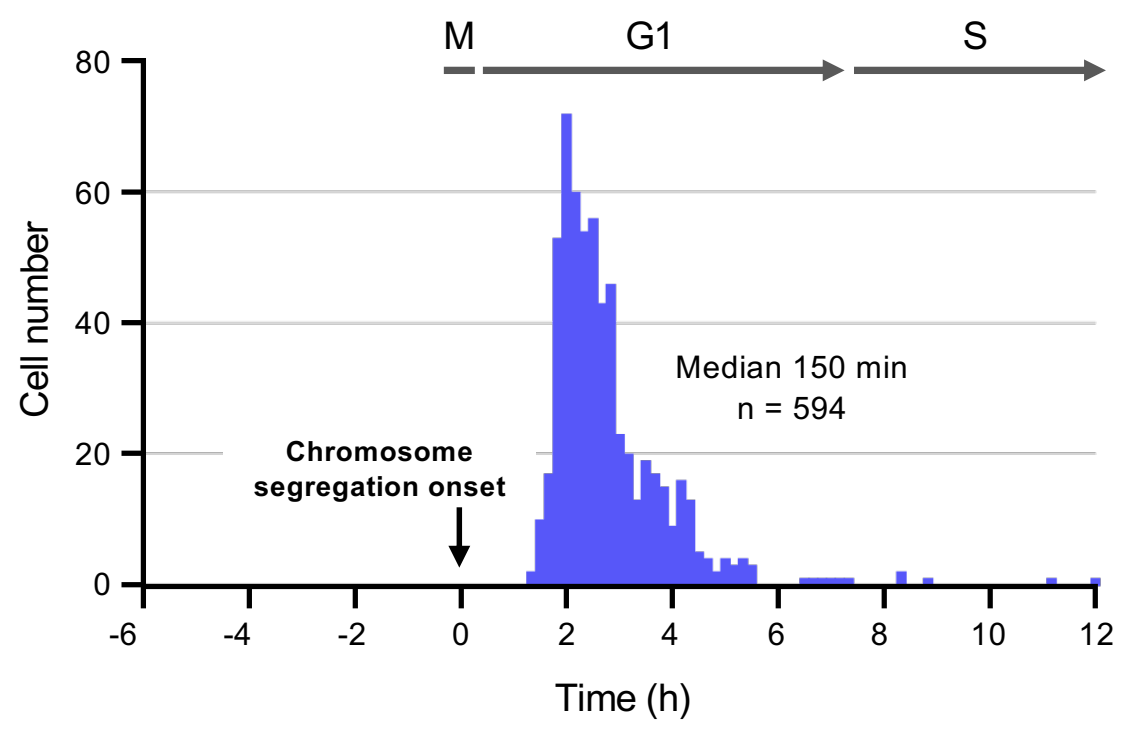

Fig. 1. Gene expression from foreign DNA occurs after mitosis. 
a) Mitotic behavior

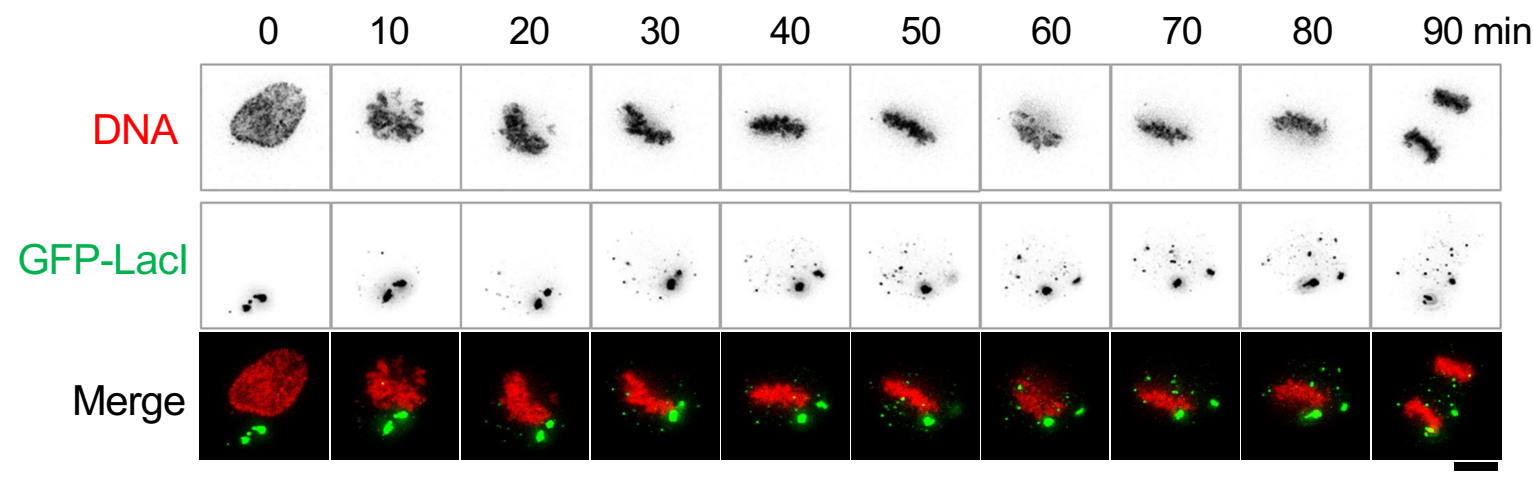

b)

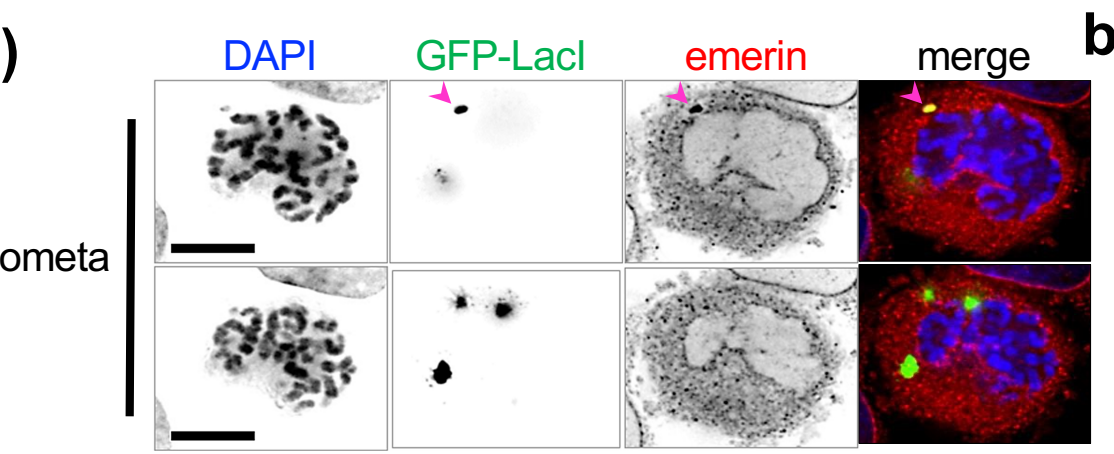

b')

c)

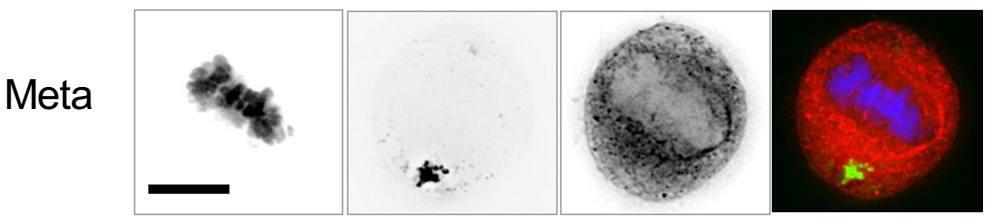

d) Depth

4.13

5.72

7.31

8.90
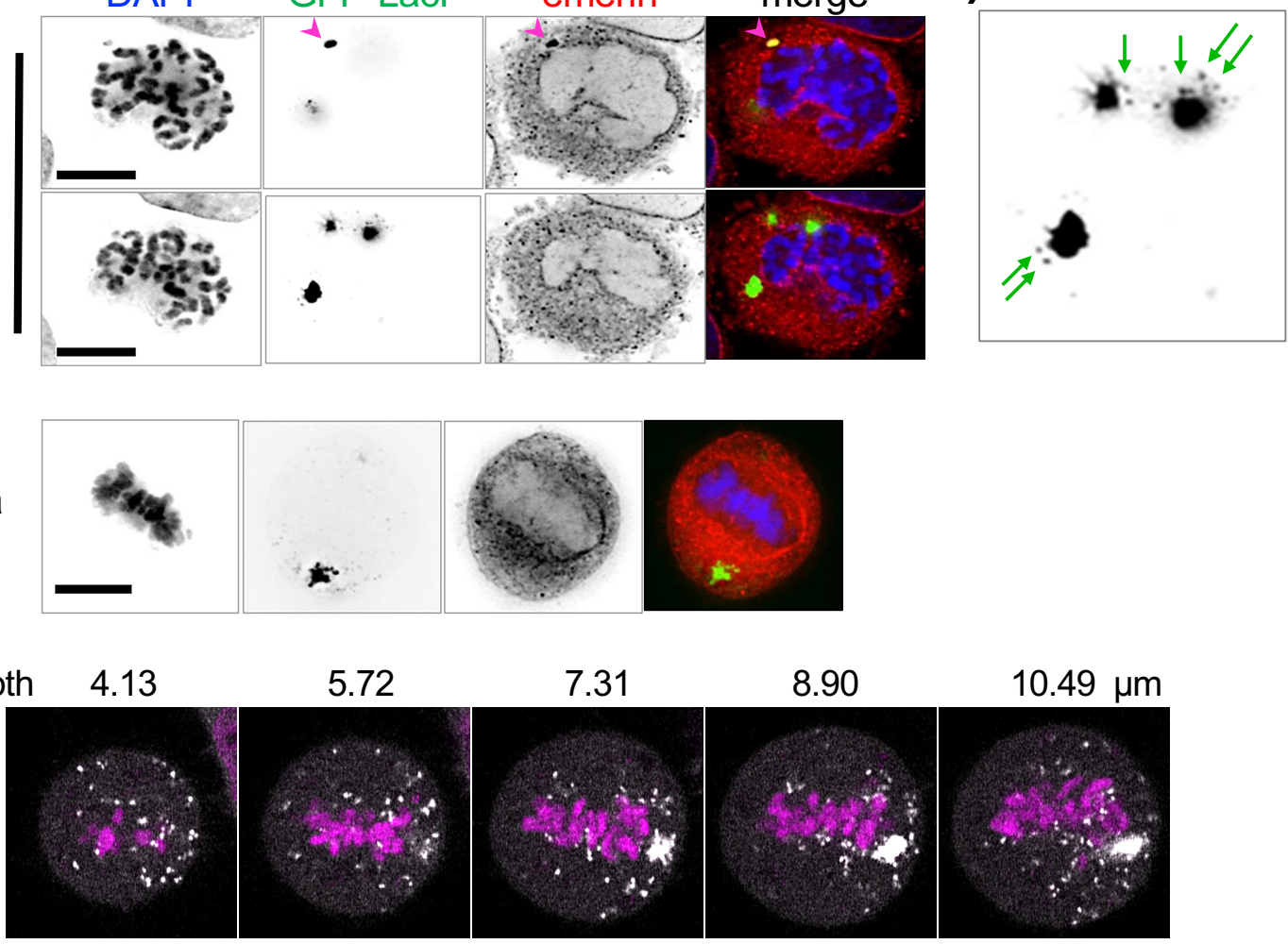

$10.49 \mu \mathrm{m}$

e)

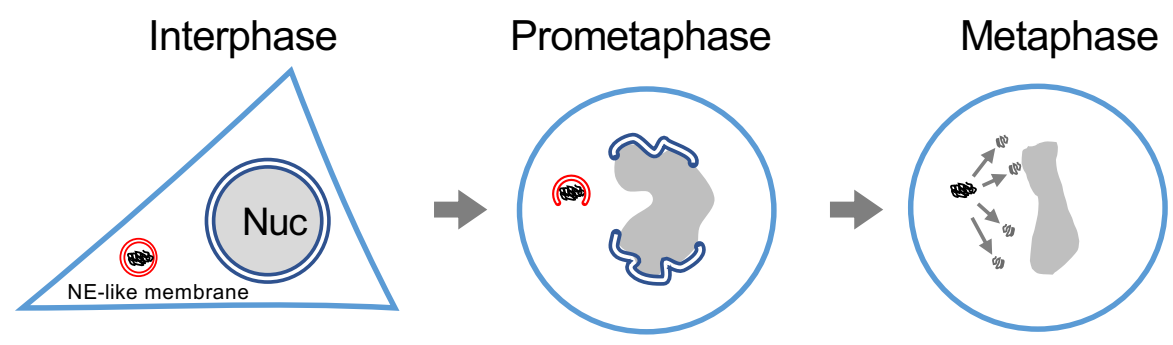

Fig. 2. DNA puncta disperse during mitosis. 
a)

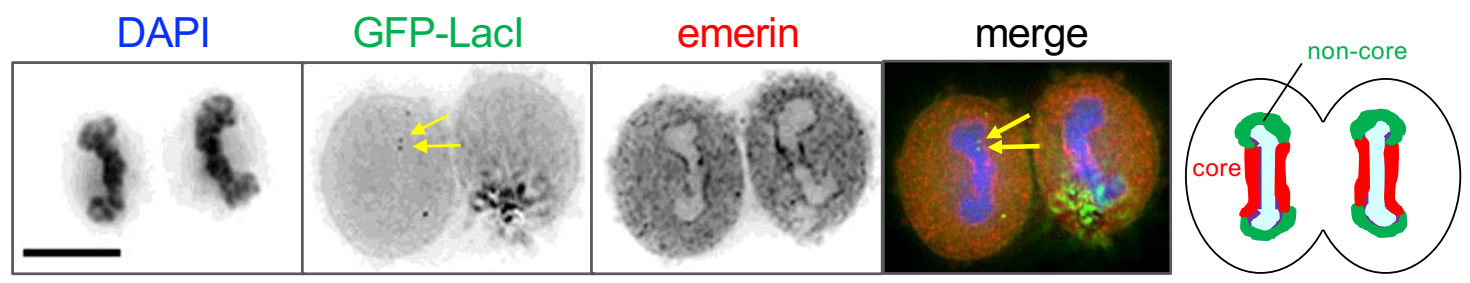

b)

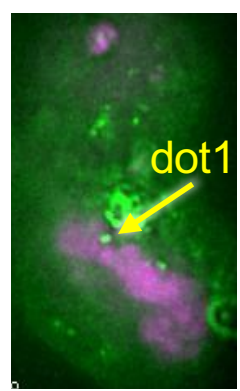

d)

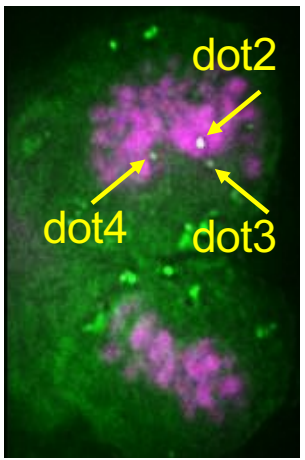

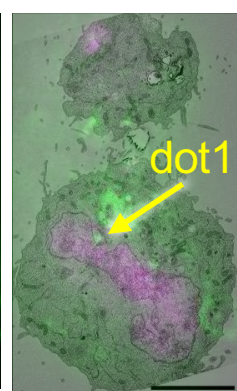

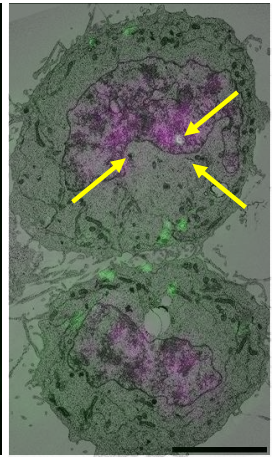

C) section 131

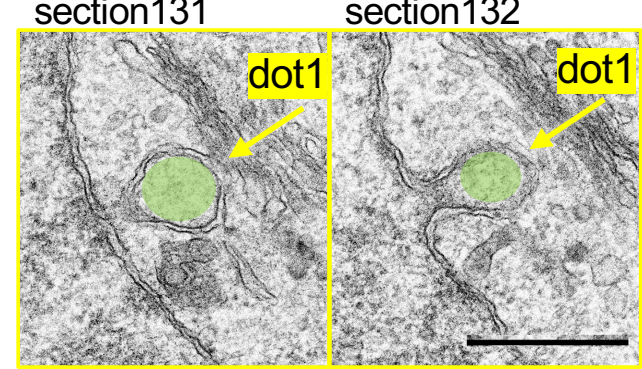

e)

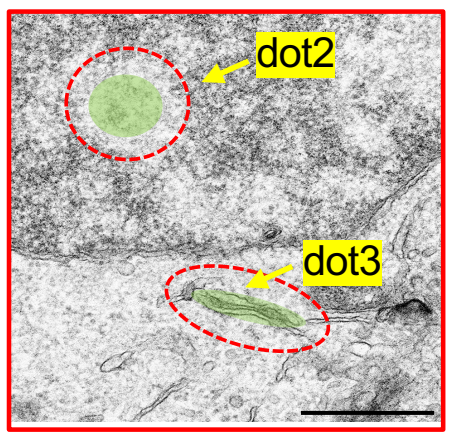

f)

\begin{tabular}{|c|c|c|c|c|}
\hline Type 1 & Type 2 & Type 3 & Type 4 & Type 5 \\
\hline $\begin{array}{c}\text { Cytoplasmic } \\
\text { circle }\end{array}$ & Sandwich & $\begin{array}{c}\text { Membrane } \\
\text { fusion }\end{array}$ & $\begin{array}{c}\text { Attached to } \\
\text { chromosome }\end{array}$ & $\begin{array}{c}\text { Inside the } \\
\text { nucleus }\end{array}$ \\
\hline Nuc & &
\end{tabular}

Fig. 3. CLEM images showing small puncta of transfected DNA in telophase cells. 
a)

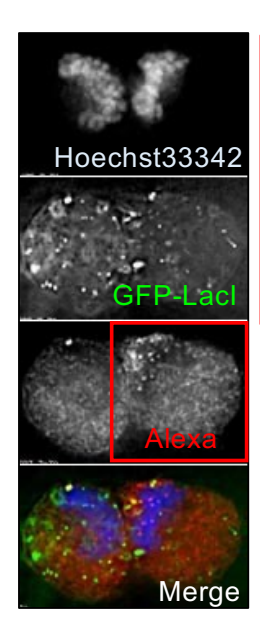

c)

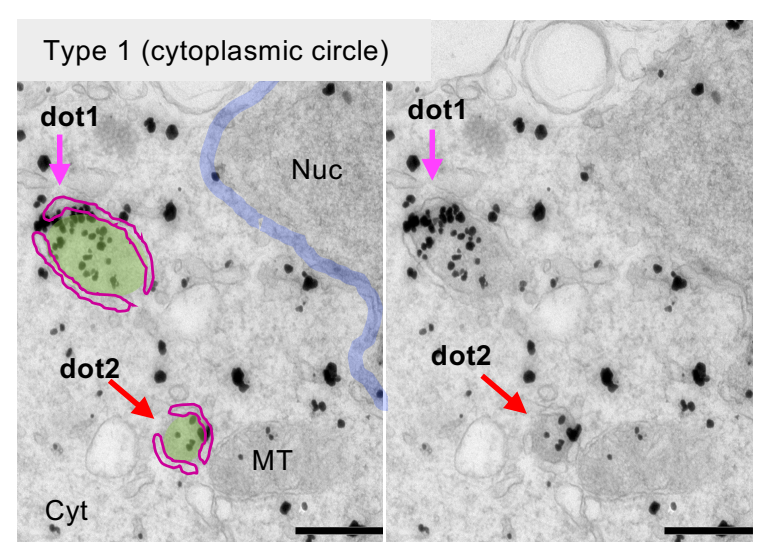

b)
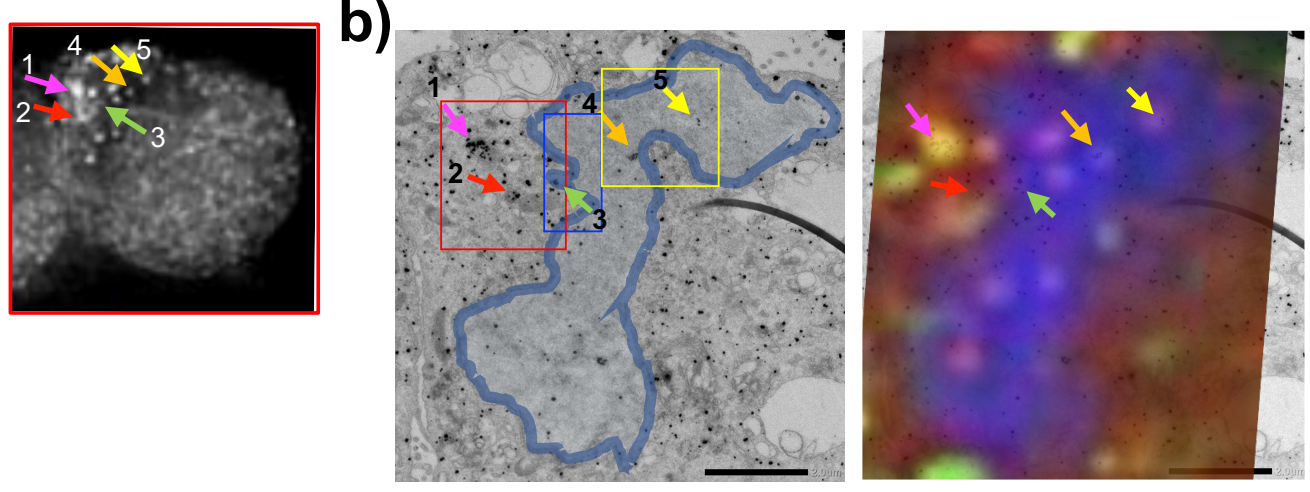

d) Type 4 or 5 (attached or inside the nucleus)

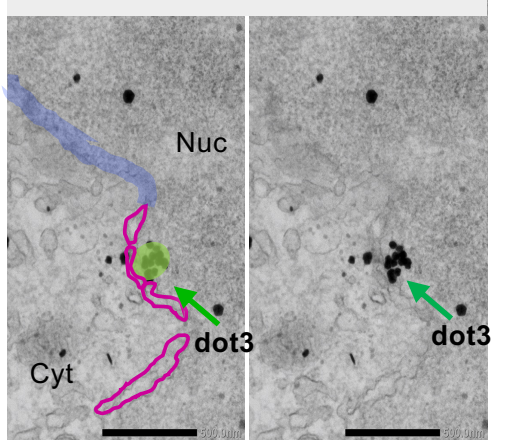

e)

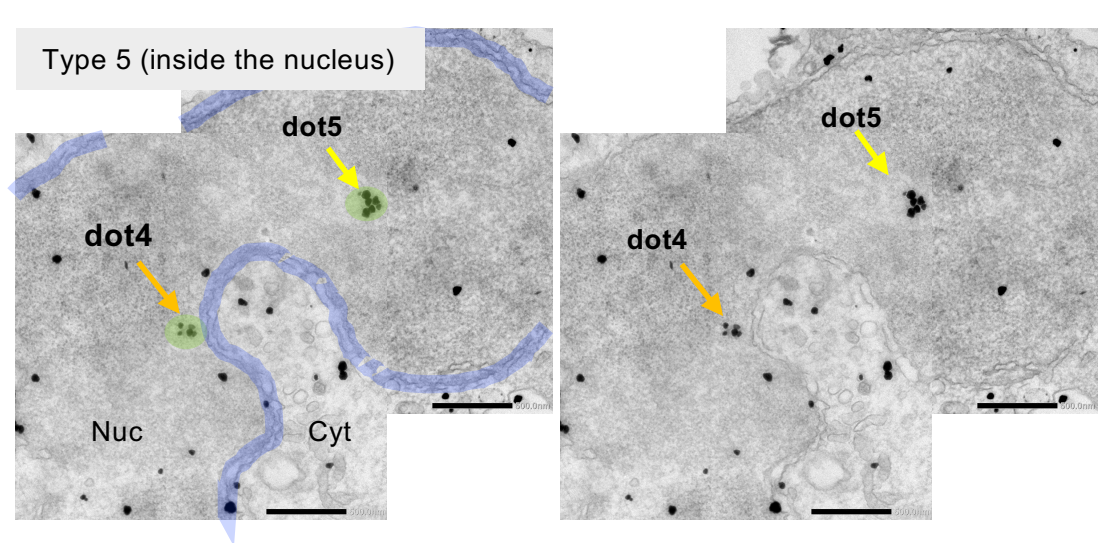

f)

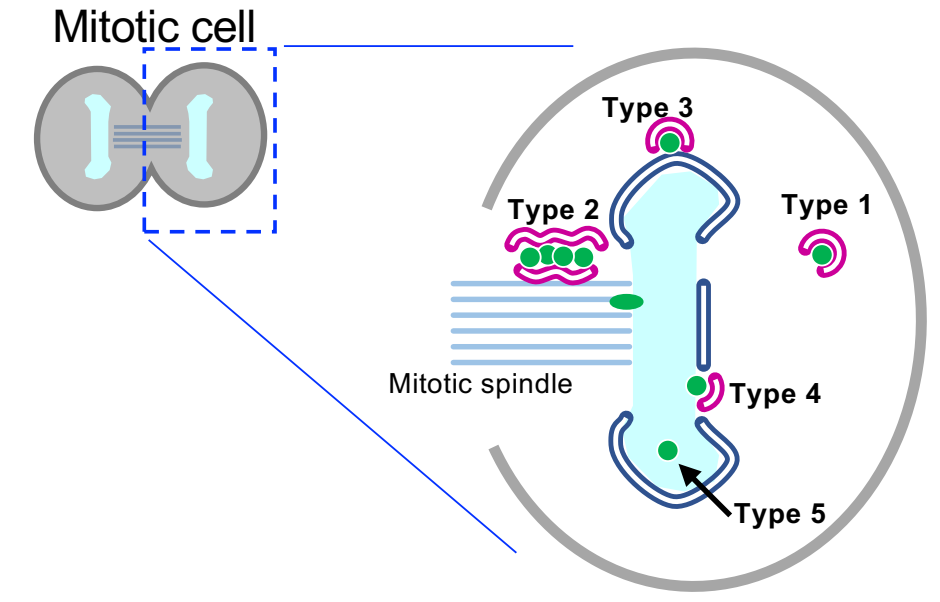

Fig. 4 Immuno-CLEM images in telophase cells 
a) Lem2 AID

Hoechst33342 RFP expression mClover3-mAID-Lem2 (max intensity projection)

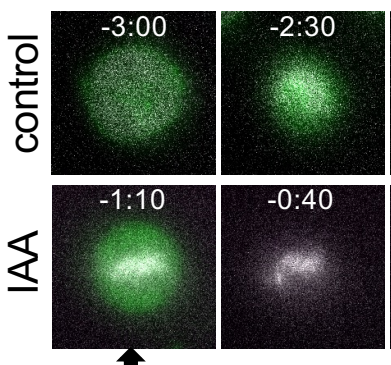

IAA addition

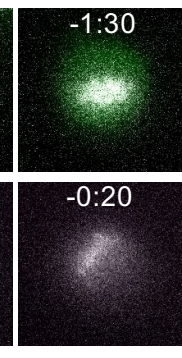

20

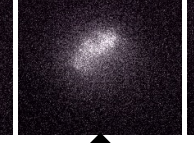

U

Chromosome segregation onset

b)

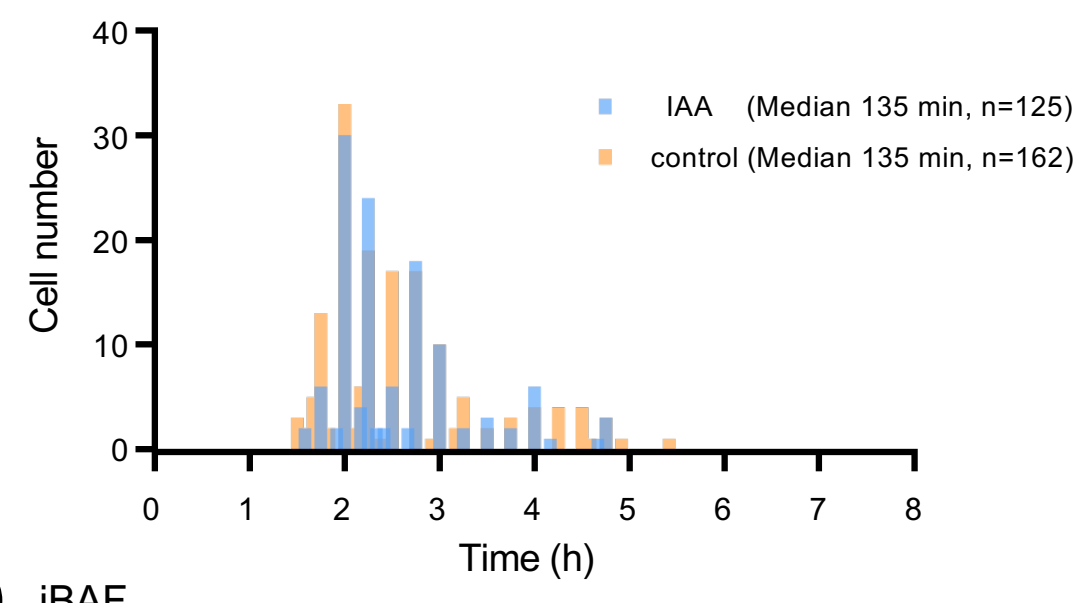

c) $\mathrm{iBAF}$

Hoechst33342 RFP expression (max intensity projection)

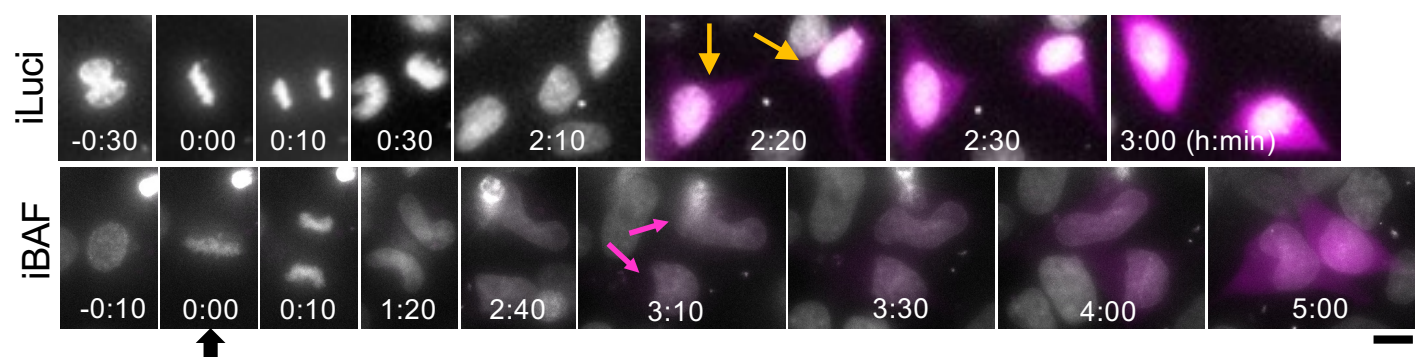

Chromosome

d) $\mathrm{iBAF}$

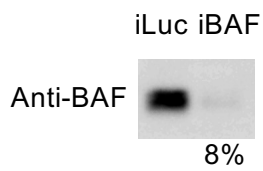

iLuc iBAF

Antitubulin e)

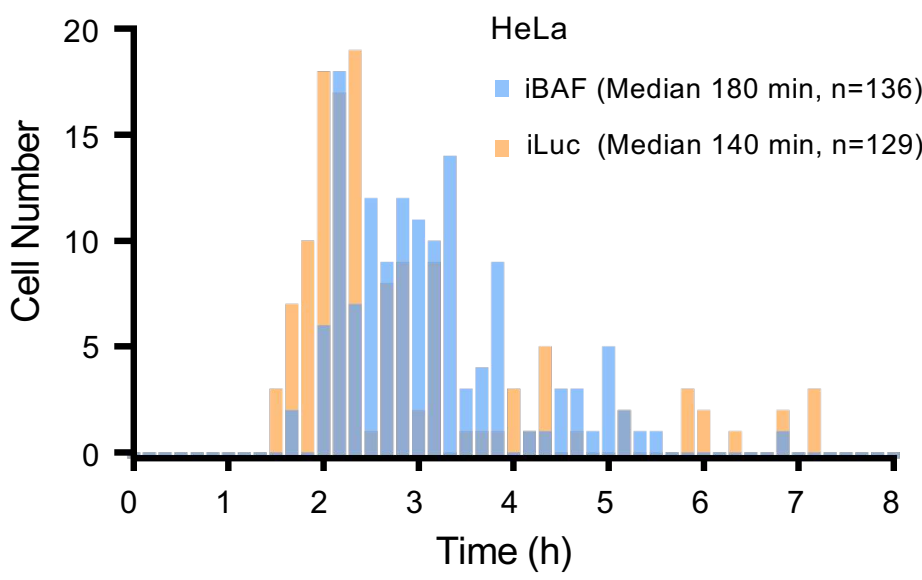

Fig. 5. Depletion of BAF delays plasmid gene expression. 
a)
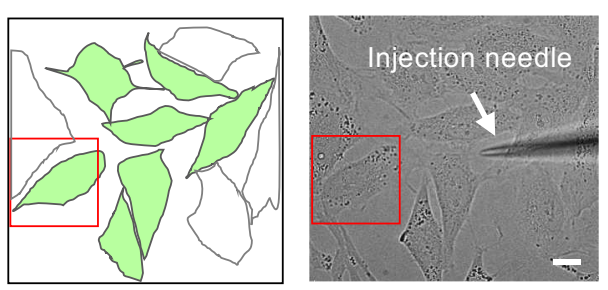

b)
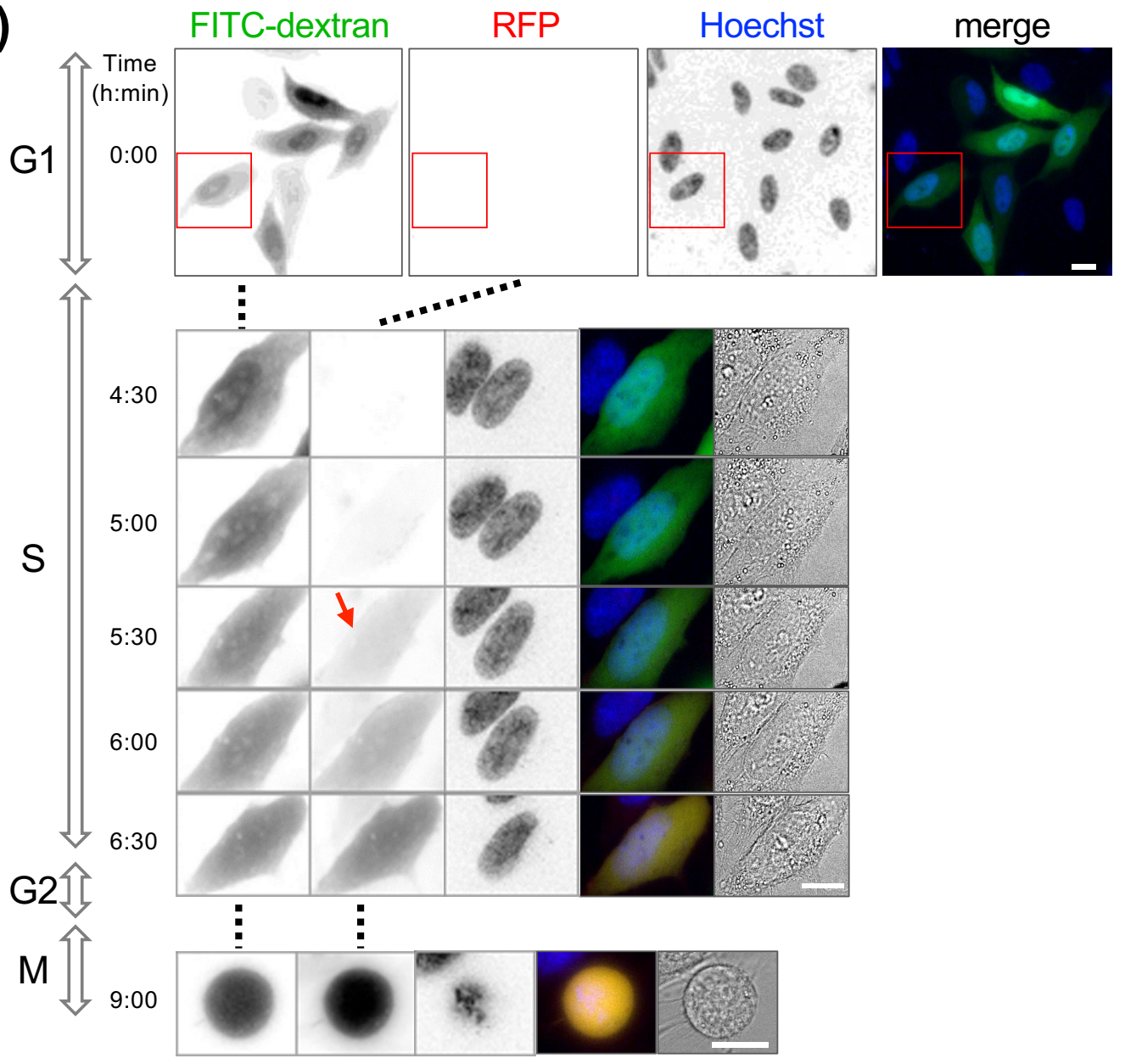

c)

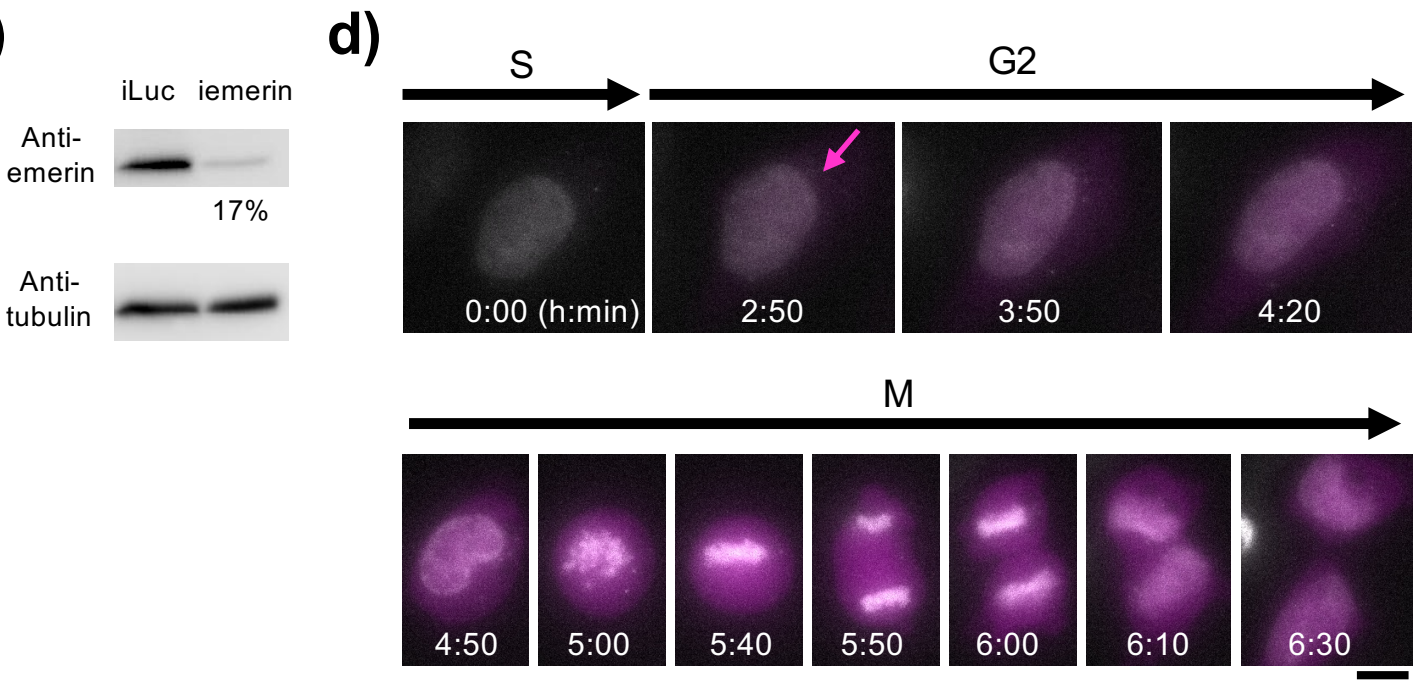

Fig. 6. NE rupture causes premitotic gene expression. 


\section{Supplementary Files}

This is a list of supplementary files associated with this preprint. Click to download.

- HaraguchiSupfinal.pdf

- SourceSupFig1U20S.xlsx

- SourceFig1dTimeExpRFP.xlsx

- SourceFig5bTimeExpAIDLem2.xIsx

- SourceFig5eTimeExpiBAF.xlsx

- SourceSupFig6bGFPiBAF.xlsx

- SourceSupFig6eiemerin.xlsx

- Haraguchisupmovie1.mov

- Haraguchisupmovie2.avi

- Haraguchisupmovie3.mov

- Haraguchisupmovie4.mov

- Haraguchisupmovie5.mov 